\title{
Two Novel 1,2,4,5-Tetrazines that Participate in Inverse Electron Demand Diels-Alder Reactions with an Unexpected Regioselectivity
}

\author{
Akiyuki Hamasaki, Richard Ducray, and Dale L. Boger \\ Department of Chemistry and The Skaggs Institute for Chemical Biology, The Scripps Research \\ Institute, 10550 North Torrey Pines Road, La Jolla, CA 92037, boger@scripps.edu
}

\begin{abstract}
Two new unsymmetrical 1,2,4,5-tetrazines, 3-methylsulfinyl-6-methylthio-1,2,4,5-tetrazine (4) and 3-(benzyloxycarbonyl)amino-6-methylsulfinyl-1,2,4,5-tetrazine (5), were prepared and scope of their participation in intermolecular inverse electron demand Diels-Alder reactions defined. As anticipated, sulfoxides 4 and $5(4>5)$ display a reactivity that is substantially greater than that of their corresponding sulfides ( $\mathbf{2}$ and $\mathbf{3}$ ) being derived from their enhanced electron-deficient character and resulting in a wider range of potential dienophile choices or the use of milder reaction conditions. The cycloaddition reactions were expectedly regioselective typically producing a single cycloadduct ensuring their synthetic utility, but both were found to proceed with a regioselectivity opposite what would be anticipated and complementary to that observed with $\mathbf{2}$ and $\mathbf{3}$.
\end{abstract}

\section{Introduction}

Electron-deficient heterocyclic azadienes have proven to be useful reagents that often participate in well-defined inverse electron demand Diels-Alder reactions with electron-rich dienophiles providing rapid access to a range of highly substituted heterocyclic systems. ${ }^{1}$ Of these, the substituted 1,2,4,5-tetrazines are the most reactive and most widely utilized heterocyclic azadienes. Typically, symmetrical 1,2,4,5-tetrazines are employed largely because of their synthetic accessibility, and synthetic studies of their utility have necessarily focused only on their relative reactivities.

In the course of our investigations of such reagents and their applications in complex natural products total synthesis, ${ }^{2-16}$ we have examined a number of such tetrazines 17,18 and introduced several new, useful symmetrical ${ }^{19}$ or unsymmetrical ${ }^{20,21} 1,2,4,5$-tetrazines. Of these, dimethyl 1,2,4,5-tetrazine-3,6-dicarboxylate (1) ${ }^{17}$ and 3,6-bis(thiomethyl)-1,2,4,5tetrazine (2) ${ }^{18}$ have been the most widely utilized of the symmetrical tetrazines, and the $N$ acyl 3-amino-6-methylthio-1,2,4,5-tetrazines (e.g., 3) ${ }^{21}$ have proven to be the most widely explored of the unsymmetrical tetrazines participating in well-behaved, effective and regioselective [4+2] cycloaddition reactions. Herein, we report the preparation of two new and useful unsymmetrical 1,2,4,5-tetrazines, 3-methylsulfinyl-6-methylthio-1,2,4,5-tetrazine (4) and 3-(benzyloxycarbonyl)amino-6-methysulfinyl-1,2,4,5-tetrazine (5), obtained by $S$ oxidation of $\mathbf{2}$ and $\mathbf{3}$, respectively, and describe studies defining the scope of their Diels-Alder reactions, Figure 1. As anticipated, both 4 and $5(4>5)$ display a reactivity that is greater than that of either $\mathbf{2}$ or $\mathbf{3}$ being derived from their enhanced electron-deficient character resulting in wider range of potential dienophile choices and/or the use of milder reaction conditions for the 
[4+2] cycloaddition reactions. Moreover, the cycloaddition reactions were expectedly regioselective typically producing a single cycloadduct ensuring their synthetic utility. Remarkably, this regioselectivity proved opposite what one would anticipate based on simple zwitterionic models or more sophisticated FMO analysis of the [4+2] cycloaddition reactions.

\section{Results and Discussion}

\section{Preparation of 1,2,4,5-Tetrazines 4 and 5}

To our knowledge, there have been only two reports of the preparation of sulfoxide substituted $1,2,4,5$-tetrazines 22,23 and only one of these examined their [4+2] cycloaddition reactivity.

23 In these latter studies, only their intramolecular [4+2] cycloaddition reaction with tethered unactivated alkynes was examined and no studies of their intermolecular Diels-Alder reactions have been disclosed. ${ }^{23}$ Both were prepared by oxidation of the corresponding thioether enlisting either the DABCO- $\mathrm{Br}_{2}$ complex ${ }^{24}$ or oxone. ${ }^{23}$ The former proved effective for selective oxidation of $\mathbf{2}$ to provide $\mathbf{4}$ as a blood red crystalline solid in good yield ( 0.55 equiv of DABCO, 1.1 equiv of $\mathrm{Br}_{2}, \mathrm{HOAc}-\mathrm{H}_{2} \mathrm{O}-\mathrm{CH}_{2} \mathrm{Cl}_{2}, 25^{\circ} \mathrm{C}, 20 \mathrm{~h}, 52 \%$ ) along with small amounts of remaining $\mathbf{2}$, Scheme 1. Increasing the amount of oxidant increased the conversion without further increasing the yield of $\mathbf{4}$, likely due to competing over oxidation, and the use of $m$-CPBA (1.1 equiv) also provided $\mathbf{4}$, but in lower yield. In either case, the over oxidation led to unidentified water soluble byproducts easily removed in the workup. Tetrazine $\mathbf{4}$, like $\mathbf{1}$, is not stable to prolonged exposure to silica gel required of a purification, but can be isolated in pure form by washing the crude reaction product with $\mathrm{Et}_{2} \mathrm{O} /$ hexane to remove small amounts of unreacted 2 and subsequently recrystallized from EtOAc/hexane (mp $\left.73-74{ }^{\circ} \mathrm{C}\right)$.

Tetrazine 3 proved essentially unreactive toward $\mathrm{DABCO}-\mathrm{Br}_{2}$ under these conditions, but was oxidized to the corresponding sulfoxide with $m$-CPBA (1.1 equiv, $\left.\mathrm{CH}_{2} \mathrm{Cl}_{2}, 0{ }^{\circ} \mathrm{C}, 30 \mathrm{~min}, 85 \%\right)$. Like 1 and $\mathbf{4}, 5$ was not sufficiently stable to prolonged exposure to silica gel to permit purification by chromatography. However, aqueous workup of the oxidation reaction including an extraction with saturated aqueous $\mathrm{NaHCO}_{3}$ to remove reagent and $m$-chlorobenzoic acid provided tetrazine 5 as a blood red oil sufficiently pure (>95\%) for [4+2] cycloaddition studies.

\section{Diels-Alder Reactions of 4}

The tetrazine 4 exhibited superb reactivity in prototypical inverse electron demand Diels-Alder reactions and was much more reactive than the corresponding sulfide $2,{ }^{18}$ Table 1 . The reaction of 4 with enamines was essentially instantaneous at $25^{\circ} \mathrm{C}$ and other electron-rich dienophiles including ketene acetals, enol ethers, and enamides readily react smoothly at room temperature to cleanly provide the [4+2] cycloadducts. Notably, no problematic detection of intermediate unaromatized product was observed and the Diels-Alder products were isolated in uniformly high yields. Remarkably, even unactivated dienophiles including phenylacetylene $(\mathbf{6 h})$ and alkyne $6 \mathbf{k}$ reacted smoothly with $\mathbf{4}$, albeit slowly at room temperature (ca. 24-48 h), requiring higher reaction temperatures for rapid reaction $\left(100^{\circ} \mathrm{C}, 1-9 \mathrm{~h}, 80-90 \%\right)$. As such, tetrazine 4 , by virtue of its enhanced electron-deficient character, exhibits a reactivity that accommodates an unusually wide range of potential dienophiles. Moreover, the [4+2] cycloaddition reactions were regioselective typically providing a single detectable product. The only exception to this generalization was dihydrofuran (Table 1, entry 10) where a trace of the second regioisomer (5-11\%) was detected. Unexpectedly, this regioselectivity proved to be opposite what one would predict from simple zwitterionic models or FMO analysis of the [4+2] cycloaddition reaction. Although this became apparent in assessing the spectroscopic properties of the cycloaddition products (e.g., 1,2-diazine $\mathrm{C} 4-\mathrm{H}$ vs $\mathrm{C} 5$-H chemical shift), single crystal X-ray structures of $\mathbf{7 c}, \mathbf{7 f}$ and $\mathbf{7 g}$ unambiguously established their structures, Figure $2{ }^{25}$ Similarly, dienophiles $\mathbf{6 j}$ and $\mathbf{6 k}$ provided regioisomeric products clearly distinguishable as the C4-substituted (C5-H $\delta 7.36)$ and $\mathrm{C} 5$-substituted (C4-H $\delta 7.88)$ 1,2-diazines with the 
latter alkyne regioselectivity being consistent with that observed with phenylacetylene (X-ray), Scheme 2. The structure of cycloadduct $\mathbf{7 b}$, which lacks the distinguishing aryl $\mathbf{C H}$, was confirmed by comparison of its spectroscopic properties with that of the two possible cycloadducts ( $\mathbf{7 b}$ vs $\mathbf{7 l}$ ) with $\mathbf{7 l}$, but not $\mathbf{7 b}$, exhibiting a characteristic diastereotopic methylene adjacent to the sulfoxide substituent similarly observed with $\mathbf{7 h}$ vs $\mathbf{7 k}$, Scheme 3 . As such, tetrazine $\mathbf{4}$ exhibits a very useful Diels-Alder reactivity accommodating an unusually wide range of potential dienophiles, and proceeds with a reaction regioselectivity opposite what one would predict.

\section{Diels-Alder Reactions of 5}

Given the useful, but unexpected observations with tetrazine 4 , an analogous but less extensive study of the [4+2] cycloaddition reactions of tetrazine 5 was conducted, Table 2. As anticipated, the reactivity of $\mathbf{5}$, by virtue of its enhanced electron-deficient character, substantially exceeded that of $\mathbf{3}$. Not only did $\mathbf{5}$ react with enamines, ketene acetals, and enol ethers rapidly and effectively at room temperature, but even the unactivated dienophile phenylacetylene (6h) provided the [4+2] cycloadduct 9f in excellent conversion (77\%) at $25^{\circ} \mathrm{C}$ requiring only $24 \mathrm{~h}$ for complete reaction. Although tetrazine $\mathbf{5}$ was slightly less reactive than $\mathbf{4}$, requiring slightly longer reaction times and providing somewhat lower conversions, it was also found to exhibit a superb [4+2] cycloaddition reactivity. Similarly, the regioselectivity of the [4+2] cycloaddition reactions was often excellent although not always as clean as that observed with $\mathbf{3}$ or $\mathbf{4}$ and, like that of $\mathbf{4}$, was found to be opposite that anticipated. This was first evident upon examination of the spectroscopic properties of the products and confirmed by X-ray for $9 c^{25}$ or by correlation with the alternative regioisomeric products available through $S$-oxidation of the analogous cycloadducts derived from tetrazine $\mathbf{3}$, Scheme 4 . These latter studies not only further verified that tetrazines $\mathbf{3}$ and $\mathbf{5}$ proceed with opposite regioselectivities in the [4+2] cycloaddition reactions and, more surprisingly, that it is the regioselectivity of 5 that is opposite what one might predict, but they also illustrate that the analogous reactions of $\mathbf{3}$ require much more vigorous reaction conditions to conduct.

\section{Reactivity and Regioselectivity}

The regioselectivity of the cycloadditions is not consistent with the expectation that the methylsulfinyl group would control the reaction orientation by stabilizing a partial negative charge at C3 (Table 3). The dienophile addition does not follow an approach predicted by this stabilization and the complementary ability of the thiomethyl or acylamino group to stabilize a partial positive charge on C6. These intuitive predictions are supported by AM1 and MNDO computational studies where $\mathrm{C} 3$ of both $\mathbf{4}$ and $\mathbf{5}$ bear a significant partial negative charge while C6 is more electropositive. Moreover, C6 bears the largest LUMO orbital coefficient indicating it should dominate the regioselectivity by preferentially combining with the dienophile $\mathrm{C} 2$ center which possesses its largest HOMO orbital coefficient. Thus, both $\mathbf{4}$ and $\mathbf{5}$ experimentally display a [4+2] cycloaddition reaction regioselectivity opposite what one would predict based on simple zwitterionic models or FMO analysis of the reactions. Only the LUMO energy levels of the FMO analysis accurately reflect the increased reactivity of 4 and $5(4>5)$ relative to 2 and 3 .

Consequently, the origin of the reversed regioselectivity is not clear. It is possible that this is related to a destabilizing steric and/or electronic interaction of the dienophile substituents with the larger and more electronegative methylsulfinyl substituent (e.g. destabilizing $-\mathrm{NR}_{2} /$ $\mathrm{CH}_{3} \mathrm{SO}$ - interaction). In part, this may explain the lower regioselectivity typically observed with 5 versus 4 including the relative behavior seen in the reaction of 5 with the terminally substituted dienophile $\mathbf{6 b}$. However, it is also interesting to note that treatment of tetrazine $\mathbf{4}$ $\left(\mathrm{Et}_{2} \mathrm{NH}\right.$, THF, 0 to $\left.25^{\circ} \mathrm{C}\right)$ or the cycloadducts $7 \mathbf{c}, 7 \mathbf{d}$ and $7 \mathbf{e}\left(\mathrm{CH}_{3} \mathrm{ONa}, \mathrm{CH}_{3} \mathrm{OH}, 25-70{ }^{\circ} \mathrm{C}\right)$ with nucleophiles only provided products derived from displacement of the methylsulfinyl 
group and not the methylsulfide. Consequently, it is also possible that the reactions proceed by stepwise addition-cyclization reactions initiated by an analogous nucleophilic addition. Although potentially reasonable for the nucleophilic dienophiles examined, no intercepted simple addition-elimination products (no cyclization) were detected and such a stepwise reaction course is unlikely for the unactivated alkynes examined.

\section{Conclusions}

The unsymmetrical 1,2,4,5-tetrazines $\mathbf{4}$ and $\mathbf{5}$ participate in well-defined and regioselective inverse electron demand Diels-Alder reactions with a wide range of electron-rich and unactivated dienophiles providing the corresponding 1,2-diazines in excellent yields. As anticipated, their enhanced electron-deficient character relative to $\mathbf{2}$ and $\mathbf{3}$ provide dienes that react faster and/or under milder reaction conditions and with a wider range of potential dienophiles. The cycloadditions are regioselective, albeit providing products opposite what is predicted using simple zwitterionic models or FMO analysis of the [4+2] cycloaddition reaction.

\section{Experimental Section}

\section{3-Methylsulfinyl-6-methylthio-1,2,4,5-tetrazine (4)}

3,6-Bis(methylthio)tetrazine $(2,500 \mathrm{mg}, 2.87 \mathrm{mmol}$ ) was dissolved in $16 \mathrm{~mL}$ of a 5:2:1 mixture of $\mathrm{HOAc}, \mathrm{H}_{2} \mathrm{O}$ and $\mathrm{CH}_{2} \mathrm{Cl}_{2}$. DABCO-2Br 2 complex $(681 \mathrm{mg}, 1.58 \mathrm{mmol})$ was added and the mixture was stirred at room temperature for $20 \mathrm{~h}$. Water was added and the mixture extracted with $\mathrm{CH}_{2} \mathrm{Cl}_{2}$, dried $\left(\mathrm{MgSO}_{4}\right)$ and evaporated. The crude material was washed with $\mathrm{Et}_{2} \mathrm{O}$ / hexane $(1: 1,3 \times)$ providing pure tetrazine $\mathbf{4}(286 \mathrm{mg}, 52 \%$ yield $)$ as a red solid. An analytically pure sample of 4 was obtained by recrystallization from EtOAc/hexane: $\mathrm{mp} 73-74{ }^{\circ} \mathrm{C}(\mathrm{EtOAc} /$ hexane); ${ }^{1} \mathrm{H} \mathrm{NMR}\left(\mathrm{CDCl}_{3}, 500 \mathrm{MHz}\right) \delta 3.18(\mathrm{~s}, 3 \mathrm{H}), 2.81$ (s, 3H); ${ }^{13} \mathrm{C} \mathrm{NMR}\left(\mathrm{CDCl}_{3}, 125\right.$ MHz) $\delta$ 178.9, 173.0, 40.1, 13.6; IR (film) $v_{\max } 1382,1242,1122,1078,1049,961,892$ $\mathrm{cm}^{-1}$; HRMS (MALDI-FTMS) $\mathrm{m} / z 212.9878\left(\mathrm{M}+\mathrm{Na}^{+}\right.$, calculated 212.9875).

\section{6-(Benzyloxycarbonyl)amino-3-(methylsulfinyl)-1,2,4,5-tetrazine (5)}

6-(Benzyloxycarbonyl)-amino-3-methylthio-1,2,4,5-tetrazine (3,100 $\mathrm{mg}, 0.36 \mathrm{mmol}$ ) was dissolved in $4 \mathrm{~mL}$ of $\mathrm{CH}_{2} \mathrm{Cl}_{2}$ and cooled to $0{ }^{\circ} \mathrm{C}$. $m$-CPBA $(68.5 \mathrm{mg}, 0.397 \mathrm{mmol})$ was added and the mixture was stirred at $0{ }^{\circ} \mathrm{C}$ for $30 \mathrm{~min}$. Saturated aqueous $\mathrm{NaHCO}_{3}$ was added and the mixture was extracted with $\mathrm{CH}_{2} \mathrm{Cl}_{2}$, dried $\left(\mathrm{MgSO}_{4}\right)$, and evaporated to give $90 \mathrm{mg}(85 \%)$ of essentially pure tetrazine $\mathbf{5}(>95 \%)$ as a red foam: ${ }^{1} \mathrm{H} \mathrm{NMR}\left(\mathrm{CDCl}_{3}, 400 \mathrm{MHz}\right) \delta 8.91$ (br s, $1 \mathrm{H}), 7.45-7.35(\mathrm{~m}, 5 \mathrm{H}), 5.34(\mathrm{~s}, 2 \mathrm{H}), 3.16(\mathrm{~s}, 3 \mathrm{H}) ;{ }^{13} \mathrm{C} \mathrm{NMR}\left(\mathrm{CDCl}_{3}, 125 \mathrm{MHz}\right) \delta 171.9$, 160.7, 150.4, 134.6, 128.6, 128.5 (2C), 128.4 (2C), 68.5, 39.6; IR (film) $v_{\max } 3196,3031,1759$, $1557,1471,1279,1211,1179,1059,964,927,742 \mathrm{~cm}^{-1}$; HRMS (ESI-TOF) $\mathrm{m} / z 316.0466$ (M $+\mathrm{Na}^{+}$, calculated 316.0475).

\section{General procedure for cycloadditions}

Tetrazine $\mathbf{4}$ or $\mathbf{5}$ was dissolved in the reaction solvent (Table 1 and 2), the dienophile was added at room temperature and the mixture was stirred at the indicated temperature for the indicated time. After completion of the reaction, the solvent was removed and the crude material was purified by chromatography on silica gel.

\section{1-(Methylsulfinyl)-4-(methylthio)-6,7-dihydro-5H-cyclopenta[d]pyridazine (7a)}

$10 \mathrm{mg}$ of $\mathbf{4}$ yielded $11.5 \mathrm{mg}$ of $\mathbf{7 a}(96 \%$, white solid) after chromatography (0-5\% MeOH/ EtOAc): mp 92-93 ${ }^{\circ} \mathrm{C}(\mathrm{EtOAc}) ;{ }^{1} \mathrm{H} \mathrm{NMR}\left(\mathrm{CDCl}_{3}, 400 \mathrm{MHz}\right) \delta 3.44-3.30(\mathrm{~m}, 2 \mathrm{H}), 3.07(\mathrm{~s}$, $3 \mathrm{H}), 2.83(\mathrm{t}, J=7.7 \mathrm{~Hz}, 2 \mathrm{H}), 2.77(\mathrm{~s}, 3 \mathrm{H}), 2.27-2.16(\mathrm{~m}, 2 \mathrm{H}) ;{ }^{13} \mathrm{C} \mathrm{NMR}\left(\mathrm{CDCl}_{3}, 125 \mathrm{MHz}\right)$ 
$\delta$ 161.7, 160.4, 145.3, 142.3, 39.3, 30.3, 30.1, 23.3, 12.7; IR (film) $v_{\max } 1541,1427,1291$,

$1191,1058,964 \mathrm{~cm}^{-1}$; HRMS (MALDI-FTMS) $\mathrm{m} / z 229.0466\left(\mathrm{M}+\mathrm{H}^{+}\right.$, calculated 229.0464).

\section{5-Ethyl-4-methyl-3-(methylsulfinyl)-6-(methylthio)pyridazine (7b)}

$20 \mathrm{mg}$ of $\mathbf{4}$ yielded $22 \mathrm{mg}$ of $\mathbf{7 b}$ (91\%, pale yellow viscous oil) after preparative TLC (EtOAc): ${ }^{1} \mathrm{H}$ NMR $\left(\mathrm{CDCl}_{3}, 400 \mathrm{MHz}\right) \delta 3.11(\mathrm{~s}, 3 \mathrm{H}), 2.74(\mathrm{q}, J=7.5 \mathrm{~Hz}, 2 \mathrm{H}), 2.71(\mathrm{~s}, 3 \mathrm{H})$, $2.60(\mathrm{~s}, 3 \mathrm{H}), 1.18(\mathrm{t}, J=7.6 \mathrm{~Hz}, 3 \mathrm{H}) ;{ }^{13} \mathrm{C} \mathrm{NMR}\left(\mathrm{CDCl}_{3}, 100 \mathrm{MHz}\right) \delta 164.6,160.4,141.3$, 134.6, 37.1, 21.8, 13.7, 12.7, 11.2; IR (film) $v_{\max } 3460,2972,2926,1715,1652,1538,1057$ $\mathrm{cm}^{-1}$; HRMS (ESI-TOF) $\mathrm{m} / z 231.0620\left(\mathrm{M}+\mathrm{H}^{+}\right.$, calculated 231.0620).

\section{5-Ethoxy-3-(methylsulfinyl)-6-(methylthio)pyridazine (7c)}

$10 \mathrm{mg}$ of 4 yielded $10.4 \mathrm{mg}$ of $\mathbf{7 c}(85 \%$, white solid) after chromatography (0-2\% MeOH/ EtOAc gradient elution). A single-crystal X-ray structure determination 25 conducted on crystals grown from benzene unambiguously established the structure of $7 \mathrm{c}$ : $\mathrm{mp} 111.5-112.5$ ${ }^{\circ} \mathrm{C}$ (benzene); ${ }^{1} \mathrm{H}$ NMR $\left(\mathrm{CDCl}_{3}, 400 \mathrm{MHz}\right) \delta 7.31(\mathrm{~s}, 1 \mathrm{H}), 4.31(\mathrm{q}, J=7 \mathrm{~Hz}, 2 \mathrm{H}), 2.98(\mathrm{~s}, 3 \mathrm{H})$, $2.69(\mathrm{~s}, 3 \mathrm{H}), 1.54(\mathrm{t}, J=7 \mathrm{~Hz}, 3 \mathrm{H}) ;{ }^{13} \mathrm{C} \mathrm{NMR}\left(\mathrm{CDCl}_{3}, 125 \mathrm{MHz}\right) \delta 166.6,157.0,156.2,99.5$, $65.5,41.8,14.0,12.6$; IR (film) $v_{\max } 1556,1353,1193,1058,1033 \mathrm{~cm}^{-1}$; HRMS (MALDIFTMS) $m / z 233.0412\left(\mathrm{M}+\mathrm{H}^{+}\right.$, calculated 233.0413).

\section{3-(Methylsulfinyl)-6-(methylthio)pyridazine (7d)}

From ethyl vinyl ether (6d): $10 \mathrm{mg}$ of $\mathbf{4}$ yielded $9.5 \mathrm{mg}$ of $\mathbf{7 d}$ (96\%, white solid) after chromatography (EtOAc). From vinyl pyrrolidinone (6e): $10 \mathrm{mg}$ of $\mathbf{4}$ afforded $6.5 \mathrm{mg}$ of $\mathbf{7 d}$ $(66 \%)$ after chromatography (EtOAc): $\mathrm{mp} 115-117^{\circ} \mathrm{C}$ (EtOAc/hexane, lit $^{28} \mathrm{mp} 118-119^{\circ}$ C); ${ }^{1} \mathrm{H} \mathrm{NMR}\left(\mathrm{CDCl}_{3}, 500 \mathrm{MHz}\right) \delta 7.91(\mathrm{~d}, J=8.8 \mathrm{~Hz}, 1 \mathrm{H}), 7.58(\mathrm{~d}, J=8.8 \mathrm{~Hz}, 1 \mathrm{H}), 2.98(\mathrm{~s}$, 3H), $2.76(\mathrm{~s}, 3 \mathrm{H}) ;{ }^{13} \mathrm{C} \mathrm{NMR}\left(\mathrm{CDCl}_{3}, 125 \mathrm{MHz}\right) \delta 166.1,164.6,127.4,121.3,41.7,13.4$; IR (film) $v_{\max } 1557,1390,1156,1053,974,854 \mathrm{~cm}^{-1}$; HRMS (MALDI-FTMS) $\mathrm{m} / \mathrm{z} 189.0153$ $\left(\mathrm{M}+\mathrm{H}^{+}\right.$, calculated 189.0151).

\section{5-Methyl-3-(methylsulfinyl)-6-(methylthio)pyridazine (7e)}

$10 \mathrm{mg}$ of $\mathbf{4}$ yielded $9.6 \mathrm{mg}$ of $\mathbf{7 e}$ (91\%, white solid) after chromatography (EtOAc): mp 106$107{ }^{\circ} \mathrm{C}$ (EtOAc/hexane); ${ }^{1} \mathrm{H}$ NMR $\left(\mathrm{CDCl}_{3}, 300 \mathrm{MHz}\right) \delta 7.74$ (s, 1H), $2.95(\mathrm{~s}, 3 \mathrm{H}), 2.74(\mathrm{~s}$, $3 \mathrm{H}), 2.36(\mathrm{~s}, 3 \mathrm{H}) ;{ }^{13} \mathrm{C} \mathrm{NMR}\left(\mathrm{CDCl}_{3}, 125 \mathrm{MHz}\right) \delta 165.4,164.8,138.5,121.0,41.7,18.5,13.4$; IR (film) $v_{\max } 1558,1347,1062 \mathrm{~cm}^{-1}$; HRMS (MALDI-FTMS) $\mathrm{m} / \mathrm{z} 203.0308\left(\mathrm{M}+\mathrm{H}^{+}\right.$, calculated 203.0307).

\section{3-(Methylsulfinyl)-6-(methylthio)-5-phenylpyridazine (7f)}

From 1-phenyl-1-(trimethylsilyloxy)ethylene (6g): $10 \mathrm{mg}$ of $\mathbf{4}$ yielded $13 \mathrm{mg}$ of $\mathbf{7 f}(94 \%$, white solid) after chromatography (60\% EtOAc/hexane). From phenylacetylene (6h): $50 \mathrm{mg}$ of 4 yielded $63 \mathrm{mg}$ of $\mathbf{7 f}(90 \%)$ after chromatography (60\% EtOAc/hexane). A single-crystal Xray structure determination 25 conducted on crystals grown from acetone $/ \mathrm{H}_{2} \mathrm{O}$ unambiguously established the structure of 7f: $\mathrm{mp} 143.2-143.8^{\circ} \mathrm{C}\left(\right.$ acetone/ $\left.\mathrm{H}_{2} \mathrm{O}\right) ;{ }^{1} \mathrm{H} \mathrm{NMR}\left(\mathrm{CDCl}_{3}, 400 \mathrm{MHz}\right)$ $\delta 7.81(\mathrm{~s}, 1 \mathrm{H}), 7.50(\mathrm{~s}, 5 \mathrm{H}), 3.02(\mathrm{~s}, 3 \mathrm{H}), 2.70(\mathrm{~s}, 3 \mathrm{H}) ;{ }^{13} \mathrm{C} \mathrm{NMR}\left(\mathrm{CDCl}_{3}, 125 \mathrm{MHz}\right) \delta 166.0$, 163.5, 141.4, 134.3, 130.1, 128.9 (2C), 128.4 (2C), 120.7, 41.7, 14.3; IR (film) $v_{\max } 1344$, $1193,1059 \mathrm{~cm}^{-1}$; HRMS (MALDI-FTMS) $\mathrm{m} / z 265.0466\left(\mathrm{M}+\mathrm{H}^{+}\right.$, calculated 265.0464).

\section{5-(4'-Bromophenyl)-3-(methylsulfinyl)-6-(methylthio)pyridazine (7g)}

$30 \mathrm{mg}$ of $\mathbf{4}$ yielded $48 \mathrm{mg}$ of $\mathbf{7 g}$ (89\%, white solid) after chromatography (50-100\% EtOAc/ hexane). A single-crystal X-ray structure determination ${ }^{25}$ conducted on crystals grown from acetone $/ \mathrm{H}_{2} \mathrm{O}$ unambiguously established the structure of $7 \mathrm{~g}$ : $\mathrm{mp} 149-150{ }^{\circ} \mathrm{C}$ (acetone/ $\left.\mathrm{H}_{2} \mathrm{O}\right) ;{ }^{1} \mathrm{H} \mathrm{NMR}\left(\mathrm{CDCl}_{3}, 500 \mathrm{MHz}\right) \delta 7.79(\mathrm{~s}, 1 \mathrm{H}), 7.64(\mathrm{~d}, J=8.8 \mathrm{~Hz}, 2 \mathrm{H}), 7.40(\mathrm{~d}, J=8.8$ 
$\mathrm{Hz}, 2 \mathrm{H}), 3.02(\mathrm{~s}, 3 \mathrm{H}), 2.70(\mathrm{~s}, 3 \mathrm{H}) ;{ }^{13} \mathrm{C} \mathrm{NMR}\left(\mathrm{CDCl}_{3}, 125 \mathrm{MHz}\right) \delta$ 166.1, 163.1, 140.1, 133.0, 132.2 (2C), 130.0 (2C), 124.6, 120.6, 41.7, 14.2; IR (film) $v_{\max }$ 1487, 1418, 1328, 1142, 1063, $1009,843,821,753 \mathrm{~cm}^{-1}$; HRMS (ESI-TOF) $\mathrm{m} / z 342.9571\left(\mathrm{M}+\mathrm{H}^{+}\right.$, calculated 342.9569).

\section{4-(2'-Hydroxyethyl)-3-(methylsulfinyl)-6-(methylthio)pyridazine (7h)}

$10 \mathrm{mg}$ of $\mathbf{4}$ yielded $8.5 \mathrm{mg}$ of $\mathbf{7 h}$ (70\%, colorless oil) after chromatography $(0-10 \% \mathrm{MeOH} /$ EtOAc). The regioisomer (7k) was isolated as a minor product $(5-11 \%):{ }^{1} \mathrm{H} \mathrm{NMR}\left(\mathrm{CDCl}_{3}\right.$, $500 \mathrm{MHz}) \delta 7.36(\mathrm{~s}, 1 \mathrm{H}), 4.04-3.98(\mathrm{~m}, 1 \mathrm{H}), 3.87-3.82(\mathrm{~m}, 1 \mathrm{H}), 3.37-3.31(\mathrm{~m}, 1 \mathrm{H}), 3.18(\mathrm{~s}$, $3 \mathrm{H}), 3.13-3.08(\mathrm{~m}, 1 \mathrm{H}), 2.74(\mathrm{~s}, 3 \mathrm{H}) ;{ }^{13} \mathrm{C} \mathrm{NMR}\left(\mathrm{CDCl}_{3}, 100 \mathrm{MHz}\right) \delta 165.1,161.7,139.7$, 128.4, 61.7, 38.9, 32.8, 13.3; IR (film) $v_{\max } 3383,1415,1353,1193,1057 \mathrm{~cm}^{-1}$; HRMS (MALDI-FTMS) $\mathrm{m} / \mathrm{z}, 233.0412\left(\mathrm{M}+\mathrm{H}^{+}\right.$, calculated 233.0413).

\section{5-(2-(tert-Butyldimethylsilyloxy)ethyl)-3-(methylsulfinyl)-6-(methylthio)pyridazine (7i)}

$10 \mathrm{mg}$ of $\mathbf{4}$ yielded $16 \mathrm{mg}$ of $\mathbf{7 i}$ (89\%, colorless oil) after chromatography (40\% EtOAc/ hexane): ${ }^{1} \mathrm{H}$ NMR $\left(\mathrm{CDCl}_{3}, 500 \mathrm{MHz}\right) \delta 7.83(\mathrm{~s}, 1 \mathrm{H}), 3.96(\mathrm{t}, J=6.2 \mathrm{~Hz}, 2 \mathrm{H}), 2.95(\mathrm{~s}, 3 \mathrm{H})$, $2.90(\mathrm{t}, J=6.2 \mathrm{~Hz}, 2 \mathrm{H}), 2.76(\mathrm{~s}, 3 \mathrm{H}), 0.84(\mathrm{~s}, 9 \mathrm{H}), 0.00(\mathrm{~s}, 6 \mathrm{H}) ;{ }^{13} \mathrm{C} \mathrm{NMR}\left(\mathrm{CDCl}_{3}, 125 \mathrm{MHz}\right)$ $\delta$ 165.5, 164.4, 139.6, 121.2, 59.6, 41.7, 35.0, 25.7, 18.1, 13.6, -5.4; IR (film) $v_{\max } 2928,1353$, 1256, 1096, 1066, 837, $777 \mathrm{~cm}^{-1}$; HRMS (MALDI-FTMS) $\mathrm{m} / z$ 347.1274 $\left(\mathrm{M}+\mathrm{H}^{+}\right.$, calculated 347.1278).

\section{4-(2-(tert-Butyldimethylsilyloxy)ethyl)-3-(methylsulfinyl)-6-(methylthio)pyridazine (7j)}

A solution of $7 \mathbf{h}(7 \mathrm{mg}, 0.03 \mathrm{mmol})$ in DMF $(300 \mu \mathrm{L})$ was treated with imidazole $(3.4 \mathrm{mg}$, $0.05 \mathrm{mmol})$ and TBSCl $(6.7 \mathrm{mg}, 0.045 \mathrm{mmol})$. The mixture was stirred for $3 \mathrm{~h}$ at room temperature before being diluted with EtOAc and washed with water. Preparative TLC (50\% EtOAc/hexane) afforded $8 \mathrm{mg}(77 \%)$ of $\mathbf{7 j}$ as a colorless oil: ${ }^{1} \mathrm{H}$ NMR $\left(\mathrm{CDCl}_{3}, 500 \mathrm{MHz}\right) \delta$ 7.39 (s, 1H), 3.98-3.90 (m, 2H), 3.27-3.22 (m, 1H), $3.14(\mathrm{~s}, 3 \mathrm{H}), 3.12-3.09(\mathrm{~m}, 1 \mathrm{H}), 2.74(\mathrm{~s}$, $3 \mathrm{H}), 0.84$ (s, 9H), -0.01 (s, 3H), -0.02 (s, 3H); ${ }^{13} \mathrm{C} \mathrm{NMR}\left(\mathrm{CDCl}_{3}, 125 \mathrm{MHz}\right) \delta$ 164.7, 161.0, 139.3 128.5, 61.6, 37.9, 33.0, 25.7 (3C), 18.1, 13.2, -5.5 (2C); IR (film) $v_{\max } 2927,1566$, 1360, 1256, 1090, 1065, 836, $777 \mathrm{~cm}^{-1}$; HRMS (MALDI-FTMS) $\mathrm{m} / z 347.1279\left(\mathrm{M}+\mathrm{H}^{+}\right.$, calculated 347.1278).

\section{5-(2'-Hydroxyethyl)-3-(methylsulfinyl)-6-(methylthio)pyridazine (7k)}

A solution of $7 \mathbf{i}(5 \mathrm{mg})$ in $100 \mu \mathrm{L}$ of THF was treated with $\mathrm{Bu}_{4} \mathrm{NF}(1.0 \mathrm{M}$ in THF, $30 \mu \mathrm{L}, 2$ equiv) at room temperature and the mixture was stirred at $25^{\circ} \mathrm{C}$ for $1 \mathrm{~h}$. Chromatography $(5 \%$ $\mathrm{MeOH} / \mathrm{EtOAc}$ ) afforded $3.3 \mathrm{mg}$ of $\mathbf{7 k}$ (98\%, white solid): mp $110-112{ }^{\circ} \mathrm{C}$ (EtOAc); ${ }^{1} \mathrm{H}$ NMR $\left(\mathrm{CDCl}_{3}, 400 \mathrm{MHz}\right) \delta 7.88(\mathrm{~s}, 1 \mathrm{H}), 4.06(\mathrm{t}, J=6.2 \mathrm{~Hz}, 2 \mathrm{H}), 2.99(\mathrm{~s}, 3 \mathrm{H}), 2.95(\mathrm{t}, J=6.2 \mathrm{~Hz}$, 2H), 2.77 (s, 3H); ${ }^{13} \mathrm{C} \mathrm{NMR}\left(\mathrm{CDCl}_{3}, 75 \mathrm{MHz}\right) \delta 165.4,164.7,139.4,120.8,59.3,41.6,34.5$, 13.7; IR (film) $v_{\max } 3377,1567,1413,1362,1193,1149,1057,958 \mathrm{~cm}^{-1}$; HRMS (MALDIFTMS) $m / z 233.0412\left(\mathrm{M}+\mathrm{H}^{+}\right.$, calculated 233.0413).

\section{4-Ethyl-5-methyl-3-(methylsulfinyl)-6-(methylthio)pyridazine (7I)}

A solution of 8 (46 mg, $0.215 \mathrm{mmol})$ in $\mathrm{CH}_{2} \mathrm{Cl}_{2}(1 \mathrm{~mL})$ was treated with $m$-CPBA (70\%, 53 $\mathrm{mg}, 0.215 \mathrm{mmol}, 1$ equiv) at $0{ }^{\circ} \mathrm{C}$. The mixture was allowed to warm to room temperature over $1 \mathrm{~h}$ before being washed with saturated aqueous $\mathrm{NaHCO}_{3}$. The organic layer was dried over $\mathrm{Na}_{2} \mathrm{SO}_{4}$ and preparative TLC $\left(\mathrm{SiO}_{2}\right.$, EtOAc) afforded $7 \mathbf{b}(13 \mathrm{mg}, 0.056 \mathrm{mmol}, 26 \%$, pale yellow viscous oil) and 71 (13 mg, $0.056 \mathrm{mmol}, 26 \%$, white solid). For 7l: $\mathrm{mp} 94-96{ }^{\circ} \mathrm{C}$ (EtOAc); ${ }^{1} \mathrm{H}$ NMR $\left(\mathrm{CDCl}_{3}, 300 \mathrm{MHz}\right) \delta 3.16-2.92(\mathrm{~m}, 2 \mathrm{H}), 3.10(\mathrm{~s}, 3 \mathrm{H}), 2.72(\mathrm{~s}, 3 \mathrm{H}), 2.28$ (s, 3H), $1.24(\mathrm{t}, J=7.5 \mathrm{~Hz}, 3 \mathrm{H}) ;{ }^{13} \mathrm{C}$ NMR $\left(\mathrm{CDCl}_{3}, 100 \mathrm{MHz}\right) \delta 165.6,159.8,140.5,135.5$, 37.6, 20.7, 14.1, 13.8, 13.6; IR (film) $v_{\max } 3475,2926,1539,1294,1206,1035,952 \mathrm{~cm}^{-1}$; HRMS (ESI-TOF) $\mathrm{m} / 2,231.0629\left(\mathrm{M}+\mathrm{H}^{+}\right.$, calculated 231.0620). 


\section{4-(Benzyloxycarbonyl)amino-1-(methylsulfinyl)-6,7-dihydro-5H-cyclopenta[ $d]$ pyridazine} (9a)

$54 \mathrm{mg}$ of $\mathbf{5}$ yielded $47 \mathrm{mg}$ of $\mathbf{9 a}(77 \%$, orange film) after chromatography $(0-1 \% \mathrm{MeOH} /$ EtOAc): ${ }^{1} \mathrm{H}$ NMR $\left(\mathrm{CDCl}_{3}, 400 \mathrm{MHz}\right) \delta 7.41-7.34(\mathrm{~m}, 5 \mathrm{H}), 5.23(\mathrm{~s}, 2 \mathrm{H}), 3.38(\mathrm{t}, J=7.6 \mathrm{~Hz}$, $2 \mathrm{H}), 3.13-3.02(\mathrm{~m}, 2 \mathrm{H}), 3.00(\mathrm{~s}, 3 \mathrm{H}), 2.26-2.08(\mathrm{~m}, 2 \mathrm{H}) ;{ }^{13} \mathrm{C} \mathrm{NMR}\left(\mathrm{CDCl}_{3}, 150 \mathrm{MHz}\right) \delta$ $160.8,153.4,153.0,148.0,141.5,135.3,128.6$ (2C), 128.5, 128.2 (2C), 67.8, 39.5, 31.6, 30.4, 24.3; IR (film) $v_{\max } 3184,2960,1733,1515,1232,1047 \mathrm{~cm}^{-1}$; HRMS (ESI-TOF) $\mathrm{m} / 2$ $332.1056\left(\mathrm{M}+\mathrm{H}^{+}\right.$, calculated 332.1063).

\section{6-(Benzyloxycarbonyl)amino-5-ethyl-4-methyl-3-(methylsulfinyl)pyridazine (9b)}

$29 \mathrm{mg}$ of $\mathbf{5}$ yielded $\mathbf{9 b}\left(9.6 \mathrm{mg}, 29 \%\right.$, colorless oil) after preparative TLC $\left(\mathrm{SiO}_{2}, \mathrm{EtOAc}\right)$. The regioisomer (9j) was isolated as a minor product $\left(3.2 \mathrm{mg}, 10 \%\right.$, colorless oil). For $\mathbf{9 b}$ : ${ }^{1} \mathrm{H}$ NMR $\left(\mathrm{CDCl}_{3}, 400 \mathrm{MHz}\right) \delta 7.39-7.36(\mathrm{~m}, 5 \mathrm{H}), 5.23(\mathrm{~s}, 2 \mathrm{H}), 3.10(\mathrm{~s}, 3 \mathrm{H}), 2.76(\mathrm{q}, J=7.6 \mathrm{~Hz}, 2 \mathrm{H})$, $2.66(\mathrm{~s}, 3 \mathrm{H}), 1.14(\mathrm{t}, J=7.6 \mathrm{~Hz}) ;{ }^{13} \mathrm{C}$ NMR $\left(\mathrm{CDCl}_{3}, 100 \mathrm{MHz}\right) \delta 161.9,154.2,153.8,139.2$, 135.3, 128.6 (2C), 128.5, 128.4 (2C), 126.9, 68.0, 37.6, 20.8, 13.3, 12.2; IR (film) $v_{\max } 3209$, 2976, 1728, 1557, 1498, 1455, 1228, $1051 \mathrm{~cm}^{-1}$; HRMS (ESI-TOF) $\mathrm{m} / z 334.1221\left(\mathrm{M}+\mathrm{H}^{+}\right.$, calculated 334.1220). For minor isomer $(\mathbf{9 j}):{ }^{1} \mathrm{H} \mathrm{NMR}\left(\mathrm{CDCl}_{3}, 400 \mathrm{MHz}\right) \delta 7.77(\mathrm{~s}, 1 \mathrm{H}), 7.39$ $7.33(\mathrm{~m}, 5 \mathrm{H}), 5.21(\mathrm{~s}, 2 \mathrm{H}), 3.13-2.95(\mathrm{~m}, 2 \mathrm{H}), 3.06(\mathrm{~s}, 3 \mathrm{H}), 2.30(\mathrm{~s}, 3 \mathrm{H}), 1.26(\mathrm{t}, J=7.6 \mathrm{~Hz}$, $3 \mathrm{H}) ;{ }^{13} \mathrm{C} \mathrm{NMR}\left(\mathrm{CDCl}_{3}, 100 \mathrm{MHz}\right) \delta 161.3,155.3,153.6,145.1,135.3,133.8,128.65(2 \mathrm{C})$, 128.56, 128.3 (2C), 68.0, 38.3, 21.1, 13.9, 13.7; IR (film) $v_{\max } 3180,2973,1732,1504,1231$, $1043 \mathrm{~cm}^{-1}$; HRMS (ESI-TOF) $\mathrm{m} / z 334.1215\left(\mathrm{M}+\mathrm{H}^{+}\right.$, calculated 334.1220).

\section{6-(Benzyloxycarbonyl)amino-5-ethoxy-3-(methylsulfinyl)pyridazine (9c)}

$70 \mathrm{mg}$ of 5 yielded $27 \mathrm{mg}$ of $\mathbf{9 c}$ (34\%, white solid) after chromatography (0-10\% MeOH/ $\left.\mathrm{CH}_{2} \mathrm{Cl}_{2}\right)$ and preparative $\mathrm{TLC}\left(\mathrm{SiO}_{2}, 5 \% \mathrm{MeOH} / \mathrm{CH}_{2} \mathrm{Cl}_{2}\right)$. The regioisomer $(\mathbf{9 h})$ was isolated as a minor product (14 $\mathrm{mg}, 17 \%$, white solid). A single-crystal X-ray structure determination 25 conducted on crystals grown from $\mathrm{EtOAc} / \mathrm{CHCl}_{3}$ unambiguously established the structure of 9c. For 9c: mp $119-121{ }^{\circ} \mathrm{C}\left(\mathrm{EtOAc} / \mathrm{CHCl}_{3}\right) ;{ }^{1} \mathrm{H} \mathrm{NMR}\left(\mathrm{CDCl}_{3}, 500 \mathrm{MHz}\right) \delta$ 7.48-7.38 (m, 6H), $5.30(\mathrm{~s}, 2 \mathrm{H}), 4.29(\mathrm{q}, J=8.5 \mathrm{~Hz}, 2 \mathrm{H}), 2.96(\mathrm{~s}, 3 \mathrm{H}), 1.52(\mathrm{t}, J=8.5 \mathrm{~Hz}$, $3 \mathrm{H}) ;{ }^{13} \mathrm{C} \mathrm{NMR}\left(\mathrm{CDCl}_{3}, 150 \mathrm{MHz}\right) \delta 165.9,151.0,148.8,147.2,135.3,128.63(2 \mathrm{C}), 128.58$ (2C), 128.54, 102.6, 67.9, 65.9, 41.9, 14.1; IR (film) $v_{\max } 1731,1572,1503,1438,1221,1042$ $\mathrm{cm}^{-1}$; HRMS (MALDI-FTMS) $\mathrm{m} / \mathrm{z} 336.1016\left(\mathrm{M}+\mathrm{H}^{+}\right.$, calculated 336.1012). For minor isomer (9h): mp 144-145 ${ }^{\circ} \mathrm{C}(\mathrm{EtOAc}) ;{ }^{1} \mathrm{H} \mathrm{NMR}\left(\mathrm{CDCl}_{3}, 400 \mathrm{MHz}\right) \delta 8.59$ (br s, $\left.1 \mathrm{H}\right), 7.89$ (s, 1H), $7.42-7.36$ (m, 5H), 5.26 (s, 2H), 4.29 (q, $J=7.0 \mathrm{~Hz}, 2 \mathrm{H}), 3.00$ (s, 3H), 1.53 (t, $J=7.0 \mathrm{~Hz}$, $3 \mathrm{H}) ;{ }^{13} \mathrm{C} \mathrm{NMR}\left(\mathrm{CDCl}_{3}, 125 \mathrm{MHz}\right) \delta 157.7,157.3,152.9,152.1,135.1,128.7$ (2C), 128.6, 128.2 (2C), 98.5, 67.8, 65.5, 37.3, 14.0; IR (film) $v_{\max } 1731,1572,1512,1228,1152,1050$, $1029 \mathrm{~cm}^{-1}$; HRMS (MALDI-FTMS) $\mathrm{m} / z 336.1017\left(\mathrm{M}+\mathrm{H}^{+}\right.$, calculated 336.1012).

\section{6-(Benzyloxycarbonyl)amino-3-(methylsulfinyl)pyridazine (9d)}

$16 \mathrm{mg}$ of 5 yielded $12 \mathrm{mg}$ of $\mathbf{9 d}$ ( $75 \%$, white solid) after chromatography (10\% EtOAc/hexane): $\mathrm{mp} 155.5-155.8{ }^{\circ} \mathrm{C}\left(\right.$ EtOAc/hexane); ${ }^{1} \mathrm{H} \mathrm{NMR}\left(\mathrm{CDCl}_{3}, 500 \mathrm{MHz}\right) \delta 8.55(\mathrm{~d}, J=9.4 \mathrm{~Hz}, 1 \mathrm{H})$, 8.39 (br s, 1H), 8.16 (d, $J=9.4 \mathrm{~Hz}, 1 \mathrm{H}), 7.42-7.39(\mathrm{~m}, 5 \mathrm{H}), 5.28(\mathrm{~s}, 2 \mathrm{H}), 2.94(\mathrm{~s}, 3 \mathrm{H}) ;{ }^{13} \mathrm{C}$ NMR $\left(\mathrm{CDCl}_{3}, 125 \mathrm{MHz}\right) \delta 165.2,155.6,152.7,134.9,128.78,128.75$ (2C), 128.4 (2C), 124.9 , 118.6, 68.0, 41.9; IR (film) $v_{\max } 1721,1573,1519,1228,1056 \mathrm{~cm}^{-1}$; HRMS (MALDI-FTMS) $\mathrm{m} / \mathrm{z} 292.0758\left(\mathrm{M}+\mathrm{H}^{+}\right.$, calculated 292.0756).

\section{6-(Benzyloxycarbonyl)amino-5-methyl-3-(methylsulfinyl)pyridazine (9e)}

$77 \mathrm{mg}$ of 5 yielded $43 \mathrm{mg}$ of $\mathbf{9 e}$ (54\%, colorless oil) after chromatography (0-10\% EtOAc/ hexane). The regioisomer (9i) was isolated as a minor product $(5.5 \mathrm{mg}, 7 \%$, white solid). For 9e: ${ }^{1} \mathrm{H}$ NMR $\left(\mathrm{CDCl}_{3}, 400 \mathrm{MHz}\right) \delta 8.01(\mathrm{~s}, 1 \mathrm{H}), 7.76(\mathrm{br} \mathrm{s}, 1 \mathrm{H}), 7.42-7.35(\mathrm{~m}, 5 \mathrm{H}), 5.24(\mathrm{~s}$, 2H), $2.94(\mathrm{~s}, 3 \mathrm{H}), 2.45$ (s, 3H); ${ }^{13} \mathrm{C} \mathrm{NMR}\left(\mathrm{CDCl}_{3}, 125 \mathrm{MHz}\right) \delta 166.8,155.1$, 153.5, 136.5, 
135.1, 128.68 (2C), 128.64, 128.4 (2C), 125.8, 68.1, 41.8, 18.4; IR (film) $v_{\max } 1732,1506$, 1234, $1049 \mathrm{~cm}^{-1}$; HRMS (MALDI-FTMS) $\mathrm{m} / z 306.0913\left(\mathrm{M}+\mathrm{H}^{+}\right.$, calculated 306.0907). For minor isomer (9i): $\mathrm{mp} 139-141{ }^{\circ} \mathrm{C}(\mathrm{EtOAc}) ;{ }^{1} \mathrm{H} \mathrm{NMR}\left(\mathrm{CDCl}_{3}, 400 \mathrm{MHz}\right) \delta 8.22(\mathrm{~s}, 1 \mathrm{H}), 8.15$ (br s, 1H), 7.43-7.37 (m, 5H), 5.26 (s, 2H), 3.08 (s, 3H), 2.69 (s, 3H); ${ }^{13} \mathrm{C} \mathrm{NMR}\left(\mathrm{CDCl}_{3}, 125\right.$ MHz) $\delta 160.2$, 155.8, 152.7, 141.6, 135.0, 128.7 (2C), 128.3 (2C), 119.1, 67.9, 37.6, 17.7; IR (film) $v_{\max } 3182,2923,1733,1558,1505,1409,1224,1152,1047,744 \mathrm{~cm}^{-1}$; HRMS (MALDIFTMS) $m / z$ 306.0912 $\left(\mathrm{M}+\mathrm{H}^{+}\right.$, calculated 306.0907).

\section{6-(Benzyloxycarbonyl)amino-3-(methylsulfinyl)-5-phenylpyridazine (9f)}

From 1-phenyl-1-(trimethylsilyloxy)ethylene (6f): $9.2 \mathrm{mg}$ of $\mathbf{5}$ yielded $9.5 \mathrm{mg}$ of $\mathbf{9 f}(83 \%$, white solid) after chromatography (60-100\% EtOAc/hexane). From phenylacetylene (6g): $10.4 \mathrm{mg}$ of $\mathbf{5}$ yielded $10 \mathrm{mg}$ of $\mathbf{9 f}(77 \%)$ after preparative TLC ( $\mathrm{SiO}_{2}$, EtOAc): $\mathrm{mp} 119-121^{\circ}$ $\mathrm{C}\left(\right.$ EtOAc/hexane) ${ }^{1} \mathrm{H}$ NMR $\left(\mathrm{CDCl}_{3}, 500 \mathrm{MHz}\right) \delta 8.07(\mathrm{~s}, 1 \mathrm{H}), 7.52-7.49(\mathrm{~m}, 5 \mathrm{H}), 7.35-7.33$ $(\mathrm{m}, 3 \mathrm{H}), 7.28-7.27(\mathrm{~m}, 2 \mathrm{H}), 5.07(\mathrm{~s}, 2 \mathrm{H}), 3.03(\mathrm{~s}, 3 \mathrm{H}) ;{ }^{13} \mathrm{C} \mathrm{NMR}\left(\mathrm{CDCl}_{3}, 125 \mathrm{MHz}\right) \delta 166.7$, 152.7, 152.0, 135.5, 135.1, 133.8, 129.9, 129.5 (2C), 128.55 (2C), 128.52, 128.4 (2C), 127.5 (2C), 124.5, 67.8, 41.9; IR (film) $v_{\max } 1731,1495,1213,1045,743,697 \mathrm{~cm}^{-1}$; HRMS (MALDI-FTMS) $\mathrm{m} / \mathrm{z} 368.1064\left(\mathrm{M}+\mathrm{H}^{+}\right.$, calculated 368.1063).

\section{6-(Benzyloxycarbonyl)amino-5-(4'-bromophenyl)-3-(methylsulfinyl)pyridazine (9g)}

$33 \mathrm{mg}$ of $\mathbf{5}$ yielded $34 \mathrm{mg}$ of $\mathbf{9 g}$ (69\%, white solid) after chromatography (50-100\% EtOAc/ hexane): $\mathrm{mp} 162-163{ }^{\circ} \mathrm{C}$ (toluene/ $\left.\mathrm{CHCl}_{3}\right) ;{ }^{1} \mathrm{H} \mathrm{NMR}\left(\mathrm{CDCl}_{3}, 500 \mathrm{MHz}\right) \delta 8.07(\mathrm{~s}, 1 \mathrm{H}), 7.92$ (brs, 1H), 7.57-7.55 (m, 2H), 7.40-7.34 (m, 5H), 7.22-7.20 (m, 2H), $5.03(\mathrm{~s}, 2 \mathrm{H}), 3.00(\mathrm{~s}$, $3 \mathrm{H}) ;{ }^{13} \mathrm{C} \mathrm{NMR}\left(\mathrm{CDCl}_{3}, 125 \mathrm{MHz}\right) \delta 167.2,152.7,152.2,135.3,135.0,133.4,132.5(2 \mathrm{C})$, 128.7 (2C), 128.6, 128.5 (2C), 128.4 (2C), 124.5, 124.2, 67.9, 41.8; IR (film) $v_{\max } 3176,2960$, 1733, 1488, 1393, 1250, 1214, 1055, $751 \mathrm{~cm}^{-1}$; HRMS (MALDI-FTMS) $\mathrm{m} / z 446.0166$ (M $+\mathrm{H}^{+}$, calculated 446.0168).

\section{6-(Benzyloxycarbonyl)amino-4-ethoxy-3-(methylthio)pyridazine (10a)}

A solution of $\mathbf{3}(20 \mathrm{mg}, 0.072 \mathrm{mmol})$ in dioxane $(300 \mu \mathrm{L})$ was treated with ketene diethyl acetal (6c, $94 \mu \mathrm{L}, 10$ equiv). The mixture was heated at $100{ }^{\circ} \mathrm{C}$ in a closed vessel for $2 \mathrm{~h}$. Chromatography (30\% EtOAc/hexane) afforded $22 \mathrm{mg}$ of 10a (95\%, white solid): $\mathrm{mp} 167$ $168{ }^{\circ} \mathrm{C}\left(\right.$ EtOAc); ${ }^{1} \mathrm{H}$ NMR $\left(\mathrm{CDCl}_{3}, 400 \mathrm{MHz}\right) \delta 8.07$ (br s, $\left.1 \mathrm{H}\right), 7.59(\mathrm{~s}, 1 \mathrm{H}), 7.42-7.34(\mathrm{~m}$, $5 \mathrm{H}), 5.23(\mathrm{~s}, 2 \mathrm{H}), 4.21(\mathrm{q}, J=7.0 \mathrm{~Hz}, 2 \mathrm{H}), 2.59(\mathrm{~s}, 3 \mathrm{H}), 1.50(\mathrm{t}, J=7.0 \mathrm{~Hz}, 3 \mathrm{H}) ;{ }^{13} \mathrm{C}$ NMR $\left(\mathrm{CDCl}_{3}, 125 \mathrm{MHz}\right) \delta 155.8,153.6,153.3,150.8,135.5,128.6$ (2C), 128.4, 128.0 (2C), 96.1, 67.3, 64.7, 14.0, 12.2; IR (film) $v_{\max } 2923,1723,1589,1571,1515,1373,1357,1239,1156$, $1117,1034,750 \mathrm{~cm}^{-1}$; HRMS (MALDI-FTMS) $\mathrm{m} / z 320.1059\left(\mathrm{M}+\mathrm{H}^{+}\right.$, calculated 320.1063).

\section{6-(Benzyloxycarbonyl)amino-4-methyl-3-(methylthio)pyridazine (10b)}

A solution of $\mathbf{3}(20 \mathrm{mg}, 0.072 \mathrm{mmol})$ in dioxane $(300 \mu \mathrm{L})$ was treated with 2-methoxypropene (6e, $69 \mu \mathrm{L}, 10$ equiv). The mixture was heated in a closed vessel at $100{ }^{\circ} \mathrm{C}$ for $18 \mathrm{~h}$. Chromatography (20\% EtOAc/hexane) afforded $10 b\left(8 \mathrm{mg}, 39 \%\right.$, white solid): $\mathrm{mp} 151-152^{\circ}$ $\mathrm{C}$ (EtOAc); ${ }^{1} \mathrm{H}$ NMR $\left(\mathrm{CDCl}_{3}, 300 \mathrm{MHz}\right) \delta 7.96(\mathrm{~s}, 1 \mathrm{H}), 7.82$ (br s, $\left.1 \mathrm{H}\right), 7.43-7.35(\mathrm{~m}, 5 \mathrm{H})$, $5.23(\mathrm{~s}, 2 \mathrm{H}), 2.67(\mathrm{~s}, 3 \mathrm{H}), 2.28(\mathrm{~s}, 3 \mathrm{H}) ;{ }^{13} \mathrm{C} \mathrm{NMR}\left(\mathrm{CDCl}_{3}, 125 \mathrm{MHz}\right) \delta 158.5,153.0,152.2$, 138.3, 135.4, 128.6 (2C), 128.5, 128.2 (2C), 116.8, 67.5, 18.5, 13.1; IR (film) $v_{\max } 1721,1570$, 1502, 1232, 1149, 1110, 1041, 750, $695 \mathrm{~cm}^{-1}$; HRMS (MALDI-FTMS) $\mathrm{m} / z 290.0956$ (M $+\mathrm{H}^{+}$, calculated 290.0958).

\section{6-(Benzyloxycarbonyl)amino-5-methyl-3-(methylthio)pyridazine (10c)}

A solution of $\mathbf{3}(25 \mathrm{mg}, 0.090 \mathrm{mmol})$ in dioxane $(0.2 \mathrm{~mL})$ was treated with $1-$ morpholinopropene ${ }^{26}\left(57 \mathrm{mg}, 0.45 \mathrm{mmol}, 5\right.$ equiv). The mixture was stirred at $25^{\circ} \mathrm{C}$ for $1 \mathrm{~h}$ before solvent was removed. The residue was dissolved in $10 \% \mathrm{HOAc} /$ benzene $(0.2 \mathrm{ml})$ and 
stirred at $25{ }^{\circ} \mathrm{C}$ for $15 \mathrm{~h}$. Neutralization with saturated aqueous $\mathrm{NaHCO}_{3}$, extraction $\left(\mathrm{CH}_{2} \mathrm{Cl}_{2}\right)$ and chromatography (40\% EtOAc/hexane) affoded $10 \mathrm{c}(24 \mathrm{mg}, 92 \%$, white solid): mp $117-119^{\circ} \mathrm{C}(\mathrm{EtOAc}) ;{ }^{1} \mathrm{H} \mathrm{NMR}\left(\mathrm{CDCl}_{3}, 400 \mathrm{MHz}\right) \delta 7.53(\mathrm{~s}, 1 \mathrm{H}), 7.40-7.32(\mathrm{~m}, 5 \mathrm{H}), 7.17$ $(\mathrm{s}, 1 \mathrm{H}), 5.20(\mathrm{~s}, 2 \mathrm{H}), 2.63(\mathrm{~s}, 3 \mathrm{H}), 2.27(\mathrm{~s}, 3 \mathrm{H}) ;{ }^{13} \mathrm{C} \mathrm{NMR}\left(\mathrm{CDCl}_{3}, 100 \mathrm{MHz}\right) \delta 145.3,135.6$, 128.6 (2C), 128.3, 128.2 (2C), 67.6, 17.7, 13.2 (4 peaks are undetected); IR (film) $v_{\max } 3208$, 2926, 1727, 1497, 1239, $1101 \mathrm{~cm}^{-1}$; HRMS (ESI-TOF) $\mathrm{m} / \mathrm{z} 290.0957\left(\mathrm{M}+\mathrm{H}^{+}\right.$, calculated 290.0958).

\section{6-(Benzyloxycarbonyl)amino-4-ethyl-5-methyl-3-(methylthio)pyridazine (10d)}

A solution of 3 (23 mg, $0.083 \mathrm{mmol})$ in $\mathrm{CH}_{2} \mathrm{Cl}_{2}(0.17 \mathrm{~mL})$ was treated with 3-morpholino-2pentene ${ }^{26}\left(\mathbf{6 b}, 26 \mathrm{mg}, 0.166 \mathrm{mmol}, 2\right.$ equiv). The mixture was stirred at $25^{\circ} \mathrm{C}$ for $1.5 \mathrm{~h}$ before removal of the solvent. The residue was treated with $10 \% \mathrm{HOAc} /$ benzene $(0.2 \mathrm{~mL})$ and the mixture stirred at $25{ }^{\circ} \mathrm{C}$ for $17 \mathrm{~h}$. Neutralization with saturated aqueous $\mathrm{NaHCO}_{3}$, extraction $\left(\mathrm{CH}_{2} \mathrm{Cl}_{2}\right)$ and preparative TLC $\left(\mathrm{SiO}_{2}, 33 \%\right.$ EtOAc/hexane) afforded $10 \mathrm{~d}(21 \mathrm{mg}, 80 \%$, pale yellow oil); mp 124-126 ${ }^{\circ} \mathrm{C}\left(\mathrm{CH}_{2} \mathrm{Cl}_{2}\right) ;{ }^{1} \mathrm{H} \mathrm{NMR}\left(\mathrm{CDCl}_{3}, 400 \mathrm{MHz}\right) \delta 7.41-7.32(\mathrm{~m}, 5 \mathrm{H}), 5.20$ (s, $2 \mathrm{H}), 2.70$ (q, $J=7.6 \mathrm{~Hz}, 2 \mathrm{H}), 2.64(\mathrm{~s}, 3 \mathrm{H}), 2.23(\mathrm{~s}, 3 \mathrm{H}), 1.17$ (t, $J=7.6 \mathrm{~Hz}, 3 \mathrm{H}) ;{ }^{13} \mathrm{C}$ NMR $\left(\mathrm{CDCl}_{3}, 100 \mathrm{MHz}\right) \delta 141.6,135.8,128.5(2 \mathrm{C}), 128.3,128.2(2 \mathrm{C}), 67.6,22.3,13.5,13.3,11.5$ (4 peaks are undetected); IR (film) $v_{\max } 3170,2971,1731,1499,1237,1071 \mathrm{~cm}^{-1}$; HRMS (ESI-TOF) $\mathrm{m} / z 318.1279\left(\mathrm{M}+\mathrm{H}^{+}\right.$, calculated 318.1271).

\section{General procedure for oxidation of 8 or 10}

A solution of $\mathbf{8}$ or $\mathbf{1 0}$ in $\mathrm{CH}_{2} \mathrm{Cl}_{2}$ was treated with $m$-CPBA ( 1 equiv) at $0{ }^{\circ} \mathrm{C}$. The reaction mixture was allowed to warm to room temperature over $0.5-1 \mathrm{~h}$. Washed with saturated aqueous $\mathrm{NaHCO}_{3}$, dried $\left(\mathrm{Na}_{2} \mathrm{SO}_{4}\right)$ and chromatography afforded 7 or 9 .

\section{Supplementary Material}

Refer to Web version on PubMed Central for supplementary material.

\section{Acknowledgements}

We gratefully acknowledge the financial support of the National Institutes of Health (CA42056) and the Skaggs Institute for Chemical Biology.

\section{References}

1. Boger, DL.; Weinreb, SM. Hetero Diels-Alder Methodology in Organic Synthesis. Academic; San Diego: 1987.

2. Reviews: Boger DL. Tetrahedron 1983;39:2869.Boger DL. Chem Rev 1986;86:781.Boger DL. Chemtracts: Org Chem 1996;9:149.

3. Streptonigrin: Boger DL, Panek JS, Duff SR. J Am Chem Soc 1985;107:5745.

4. OMP: Boger DL, Coleman RS, Panek JS, Yohannes D. J Org Chem 1984;49:4405.

5. Lavendamycin: Boger DL, Duff SR, Panek JS, Yasuda M. J Org Chem 1985;50:5790.

6. PDE-I and PDE-II: Boger DL, Coleman RS. J Am Chem Soc 1987;109:2717.

7. CC-1065: Boger DL, Coleman RS. J Am Chem Soc 1988;110:1321, 4796.

8. Prodigiosin: Boger DL, Patel M. J Org Chem 1988;53:1405.

9. Trikentrin A: Boger DL, Zhang M. J Am Chem Soc 1991;113:4230.

10. Isochrysohermidin: Boger DL, Baldino CM. J Am Chem Soc 1993;115:11418.

11. Ningalin A, Lamellarin O, Lukianol A, Storniamide A, Boger DL, Boyce CW, Labroli MA, Sehon CA, Jin Q. J Am Chem Soc 1999;121:54.

12. Phomazarin: Boger DL, Hong J, Hikota M, Ishida M. J Am Chem Soc 1999;121:2471. 
13. Ningalin B: Boger DL, Soenen DR, Boyce CW, Hedrick MP, Jin Q. J Org Chem 2000;65:2479. [PubMed: 10789460]

14. Anhydrolycorinone and related alkaloids: Boger DL, Wolkenberg SE. J Org Chem 2000;65:9120. [PubMed: 11149859]

15. Roseophilin: Boger DL, Hong J. J Am Chem Soc 2001;123:8515. [PubMed: 11525659]

16. Ningalin D: Hamasaki A, Zimpleman JM, Hwang I, Boger DL. J Am Chem Soc 2005;127:10767. [PubMed: 16045367]

17. Dimethyl 1,2,4,5-tetrazine-3,6-dicarboxylate (1): Sauer J, Mielert A, Lang D, Peter D. Chem Ber 1965;98:1435.Boger DL, Coleman RS, Panek JS, Huber FX, Sauer J. J Org Chem 1985;50:5377.Boger DL, Panek JS, Patel M. Org Syn 1991;70:79.

18. 3,6-Bis(methylthio)-1,2,4,5-tetrazine (2): StrubeREOrg SynWileyNew York1963IV967Sandström J. Acta Chem Scand 1961;15:1575.Boger DL, Sakya SM. J Org Chem 1988;53:1415.

19. 3,6-Bis(3,4-dimethoxybenzoyl)-1,2,4,5-tetrazine: Soenen DR, Zimpleman JM, Boger DL. J Org Chem 2003;68:3593. [PubMed: 12713365]

20. 3-Methoxy-6-methylthio-1,2,4,5-tetrazine: Sakya SM, Groskopf KK, Boger DL. Tetrahedron Lett 1997;38:3805.

21. N-Acyl 6-amino-3-methylthio-1,2,4,5-tetrazine (e.g., 3): Boger DL, Schaum RP, Garbaccio RM. J Org Chem 1998;63:6329. [PubMed: 11672266]

22. Johnson JL, Whitney B, Werbel LM. J Heterocyclic Chem 1980;17:501.

23. Seitz G, Dietrich S, Görge L, Richter J. Tetrahedron Lett 1986;27:2747.

24. Blair LK, Baldwin J, Smith WC Jr. J Org Chem 1977;42:1817.

25. Atomic coordinates for 7c (CCDC 282318), 7f (CCDC 282319), 7g (CCDC 282321) and 9c (CCDC 282320) have been deposited with the Cambridge Crystallographic Data Centre.

26. Stork G, Brizzolara A, Landesman H, Szmuszkovic J, Terrell R. J Am Chem Soc 1963;85:207.

27. Cazeau P, Duboudin F, Moulines F, Babot O, Dunogues J. Tetrahedron 1987;43:2075.

28. Sega T, Pollak A, Stanovnik B, Tisler M. J Org Chem 1973;38:3307. 

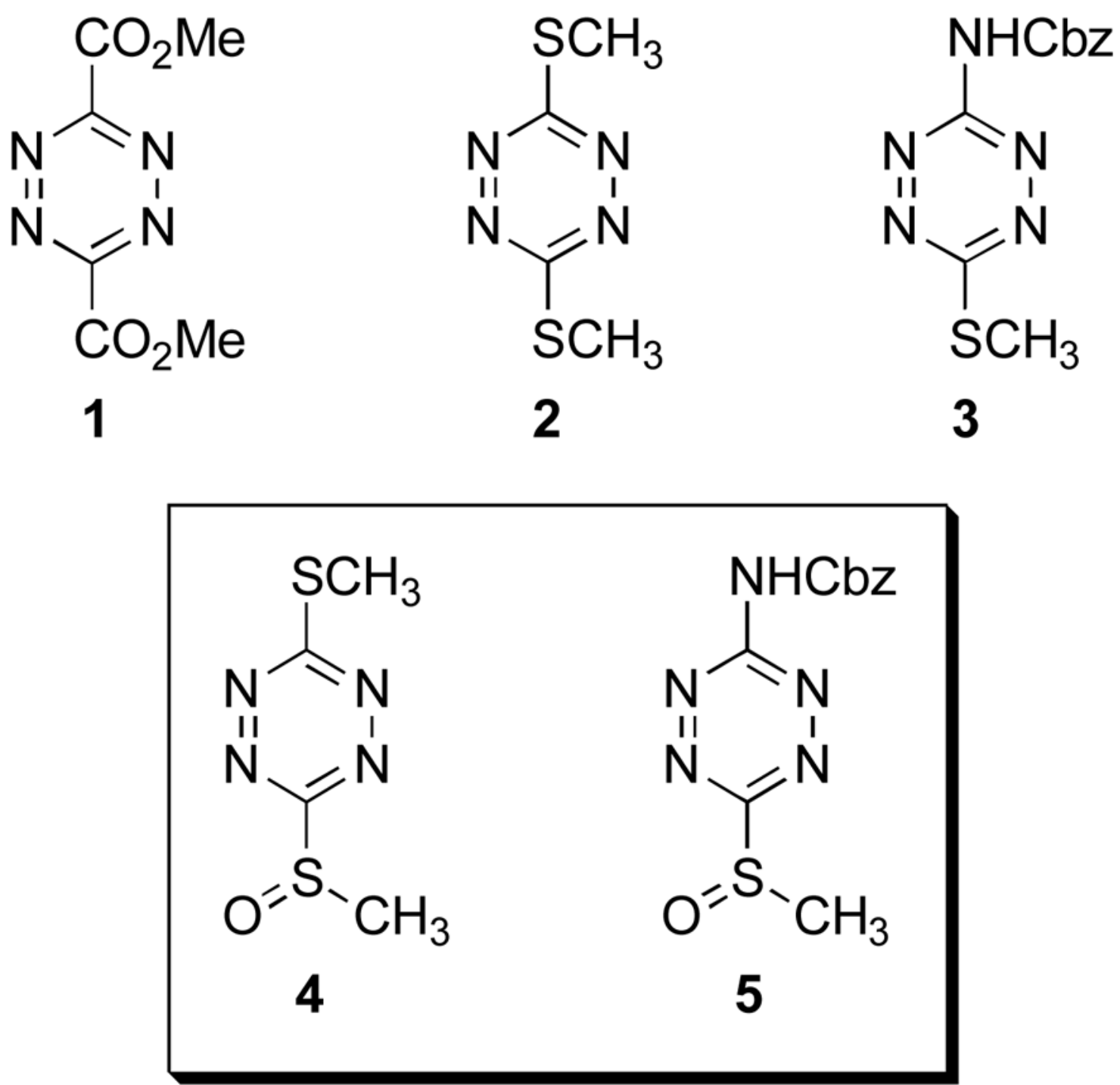

Figure 1. 

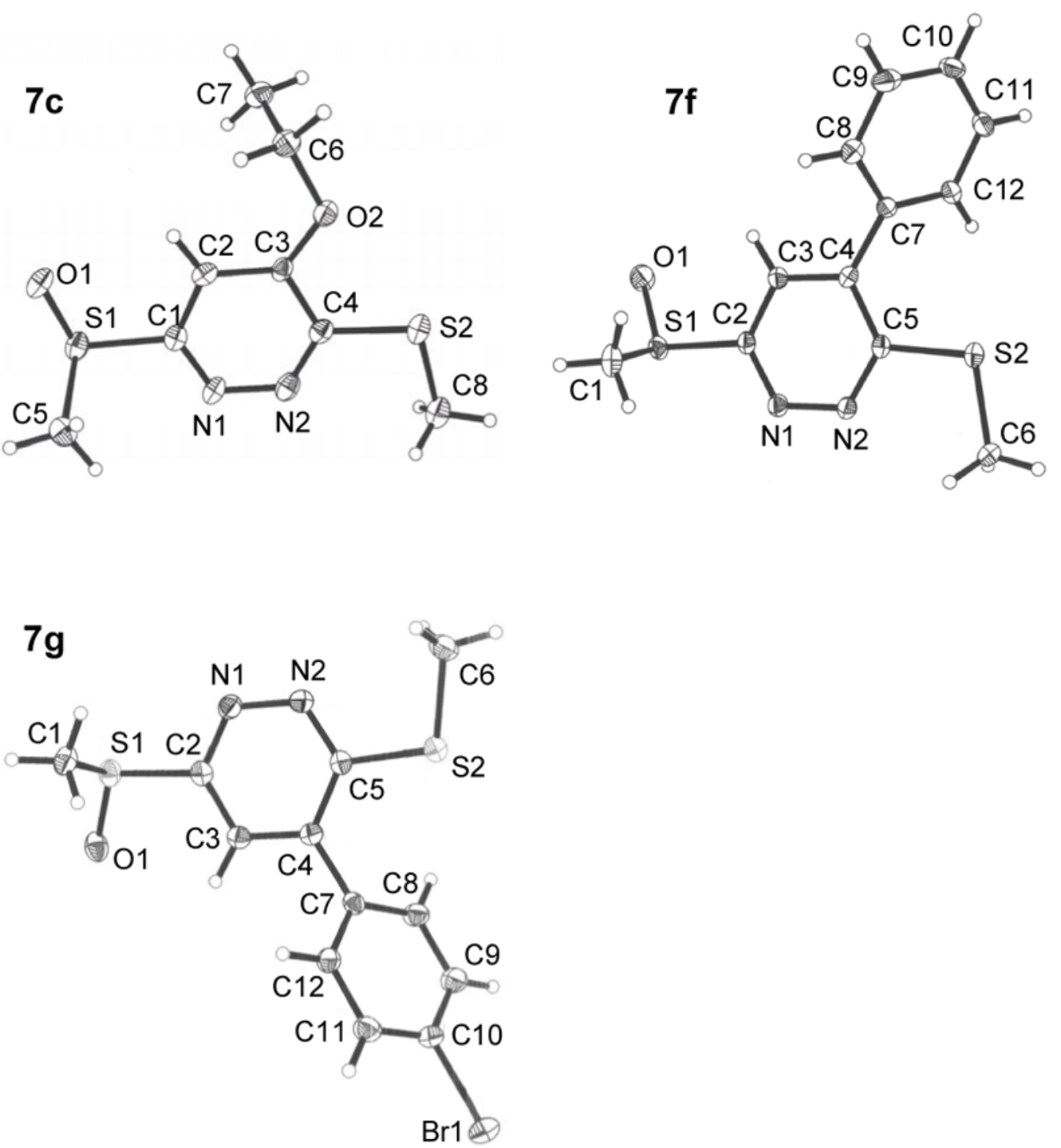

Figure 2.

ORTEP drawings of $\mathbf{7 c}, \mathbf{7 f}$ and $\mathbf{7 g}$. 


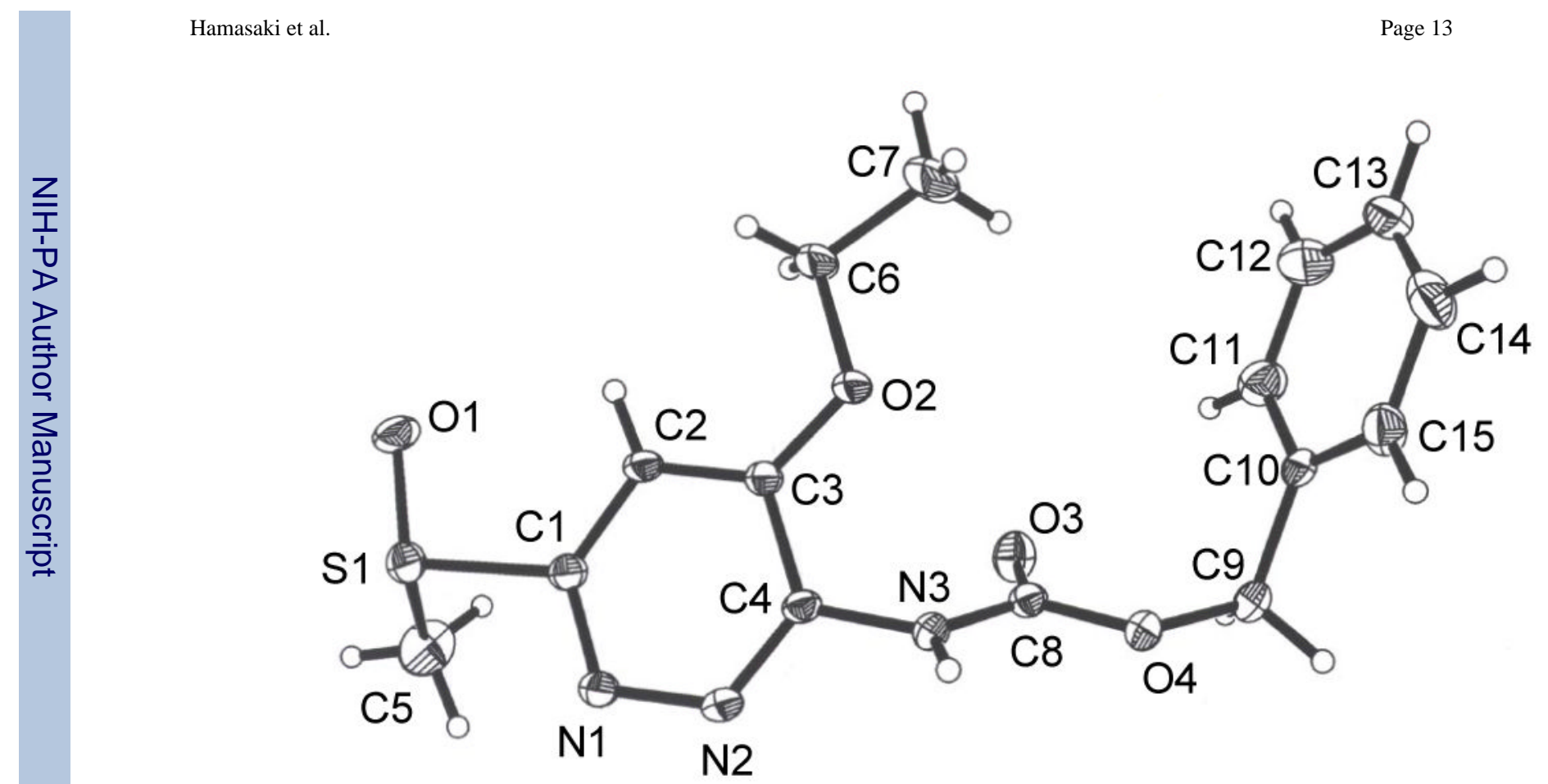

Figure 3.

ORTEP drawing of $\mathbf{9 c}$. 


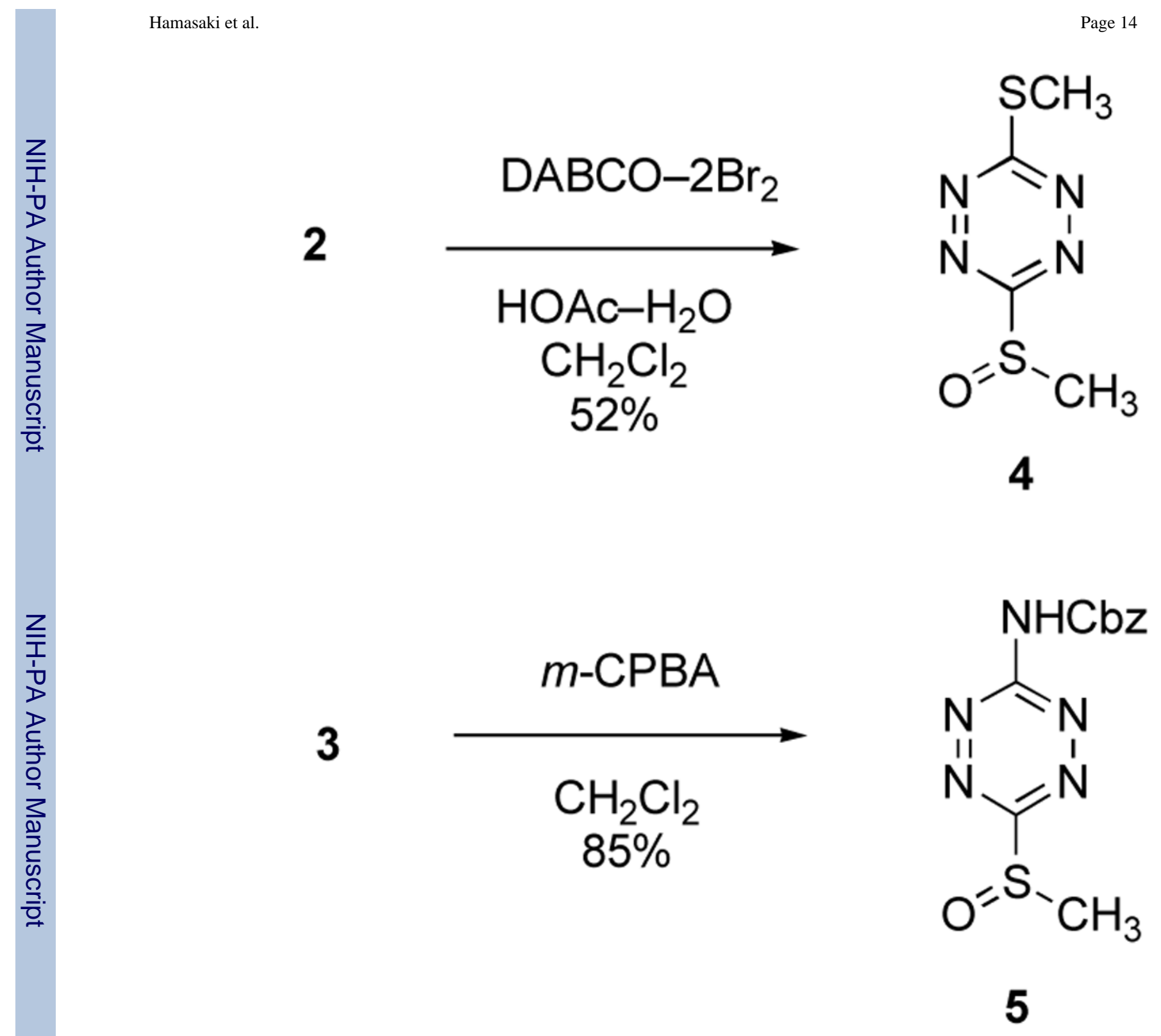

Scheme 1. 

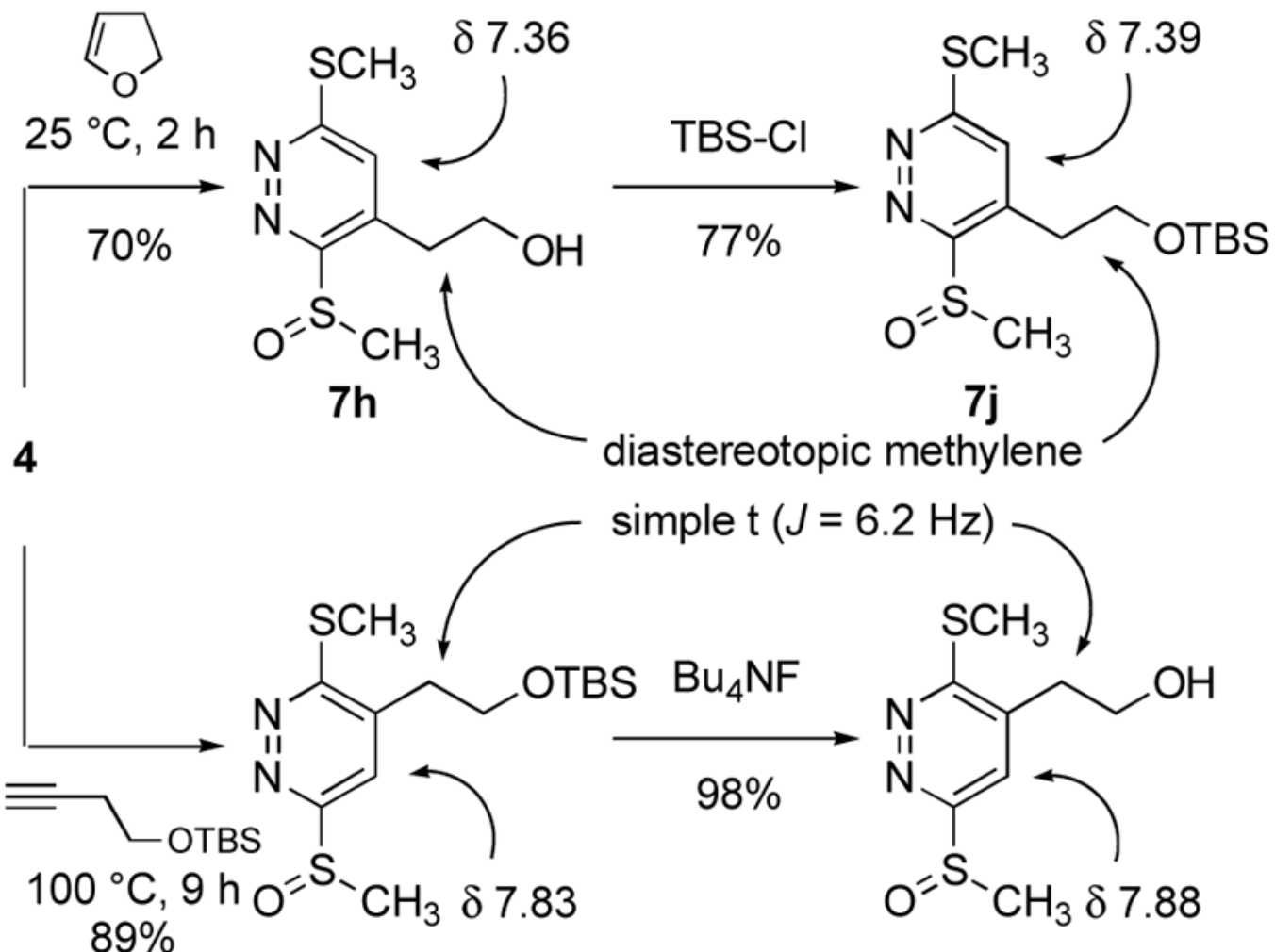
$89 \%$

$7 \mathbf{i}$

$7 k$

Scheme 2. 


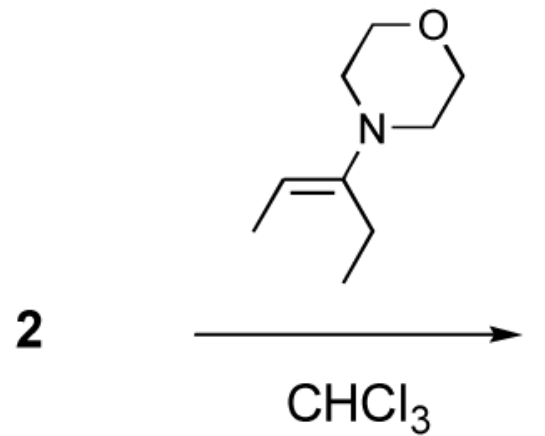

$25^{\circ} \mathrm{C}, 2 \mathrm{~h}$ and $50^{\circ} \mathrm{C}, 3 \mathrm{~h}$ then $10 \% \mathrm{HOAc} / \mathrm{C}_{6} \mathrm{H}_{6}$ $50{ }^{\circ} \mathrm{C}, 17 \mathrm{~h}$

$75 \%$

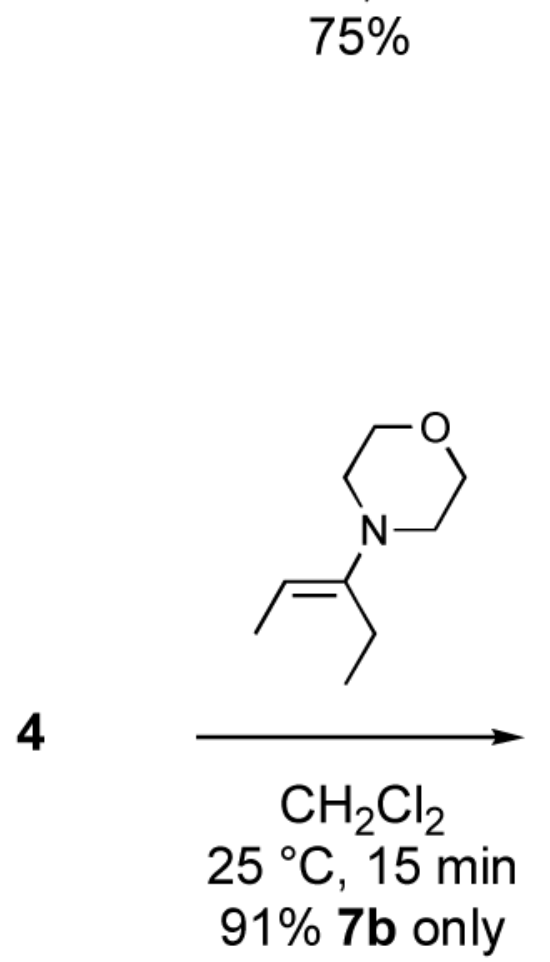

Scheme 3.
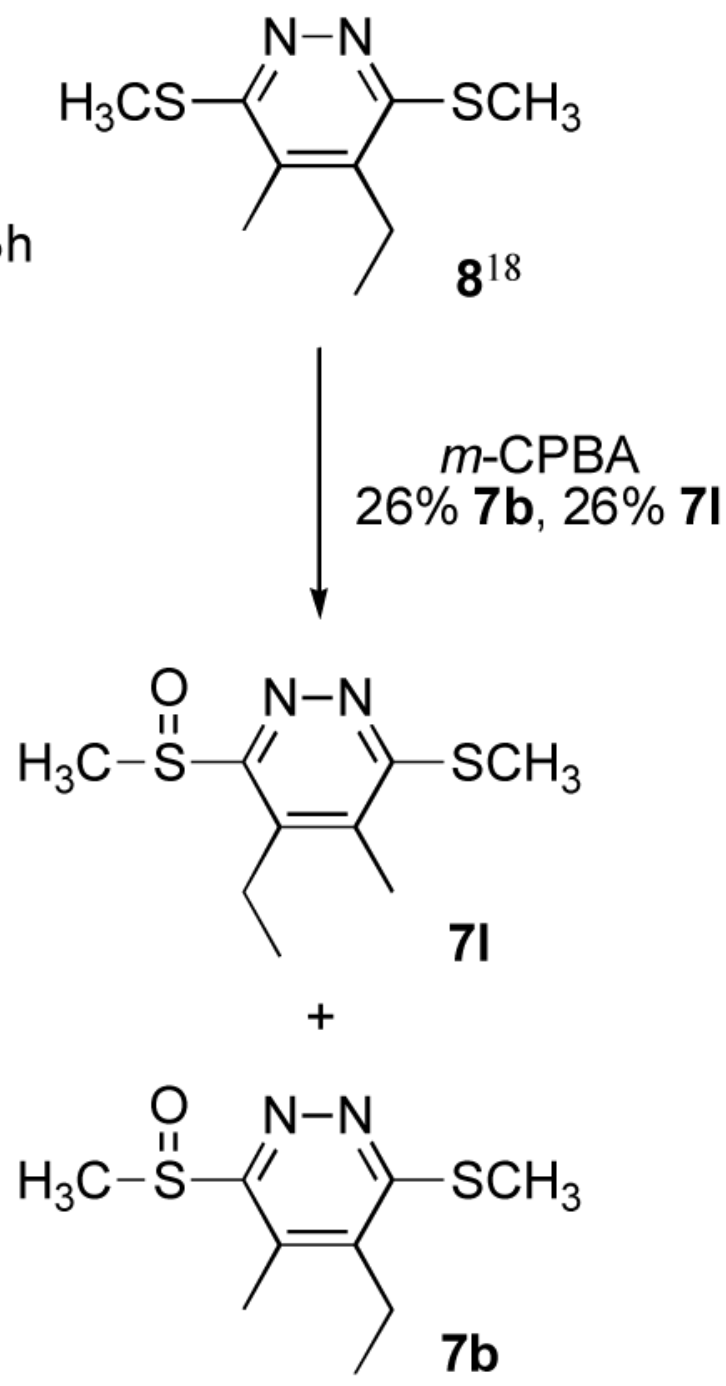
3

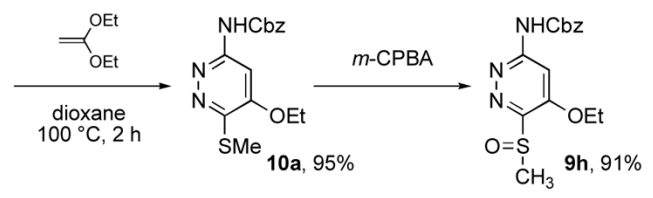

5
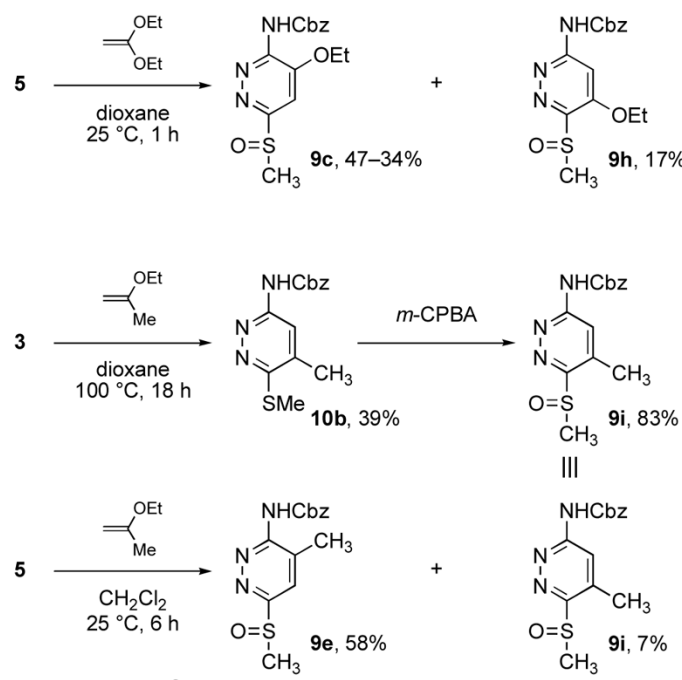

3
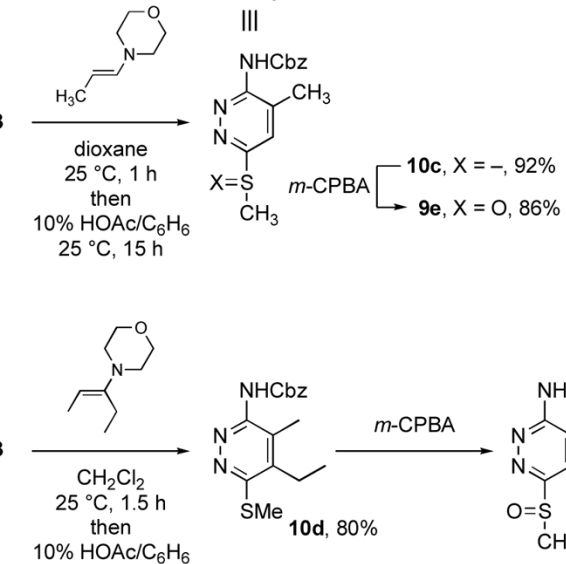
$10 \% \mathrm{HOAc} / \mathrm{C}_{6} \mathrm{H}_{6}$ $25^{\circ} \mathrm{C}, 17 \mathrm{~h}$

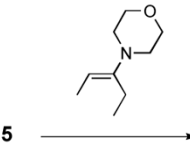
$\mathrm{CH}_{2} \mathrm{Cl}_{2}$ $25{ }^{\circ} \mathrm{C}, 15$ min

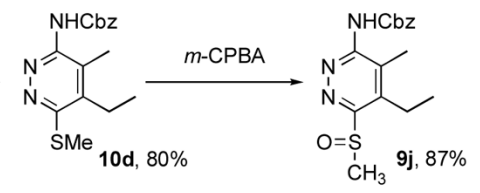

III

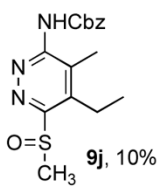

Scheme 4. 


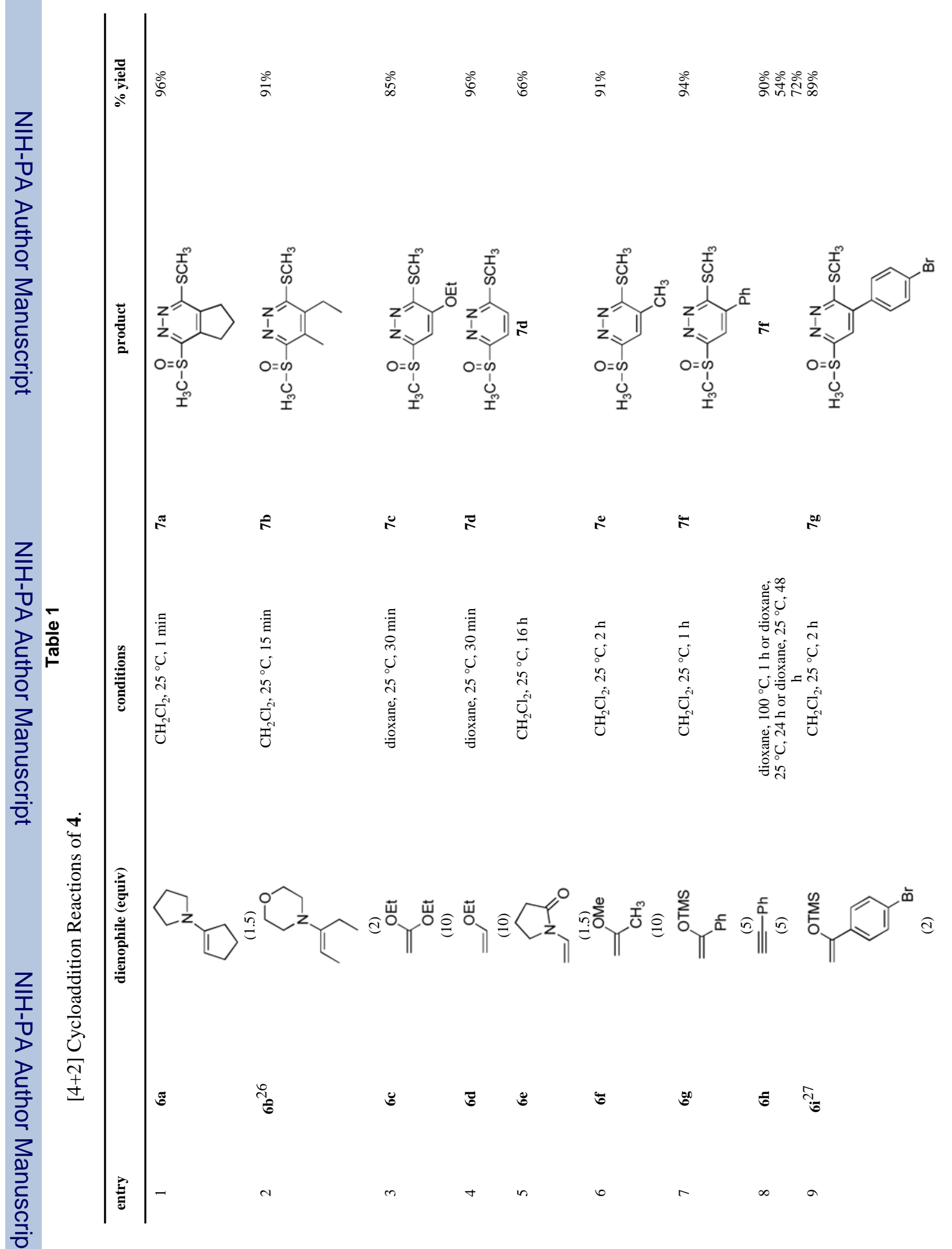


Hamasaki et al.

Page 19

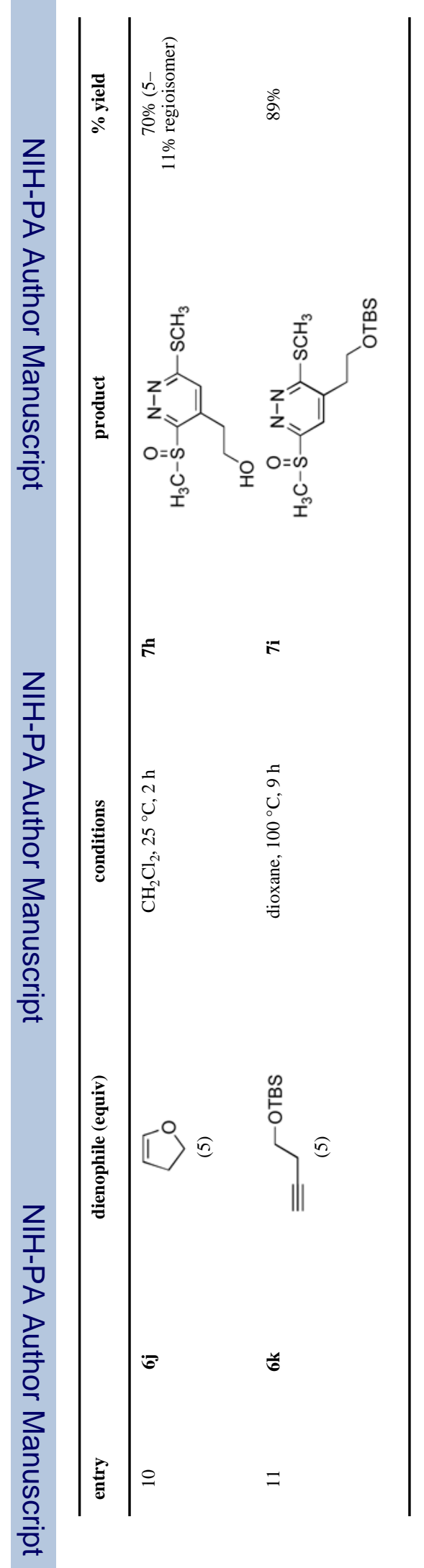

J Org Chem. Author manuscript; available in PMC 2008 August 26. 
Hamasaki et al.

Page 20

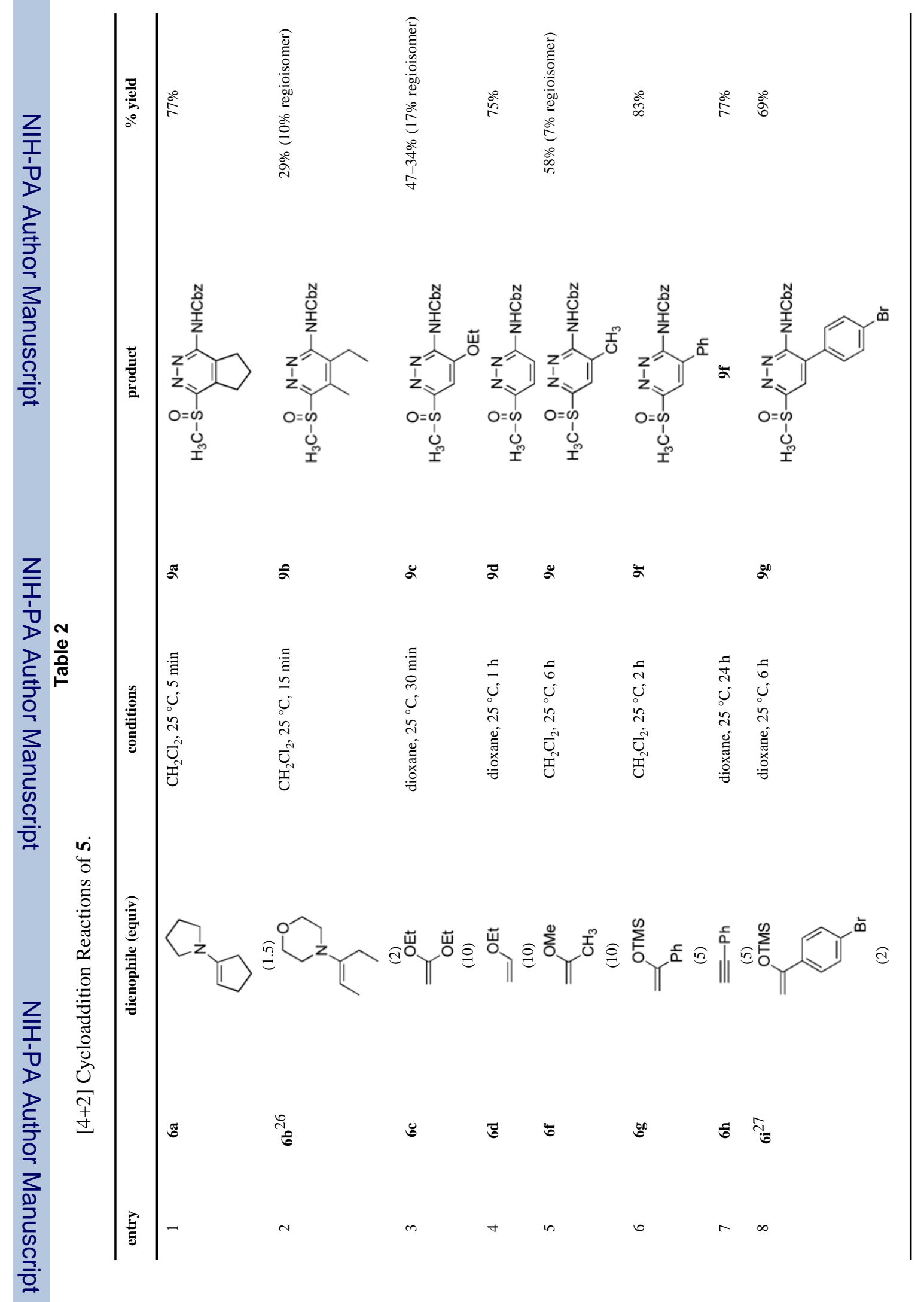

J Org Chem. Author manuscript; available in PMC 2008 August 26. 
Hamasaki et al.

Page 21

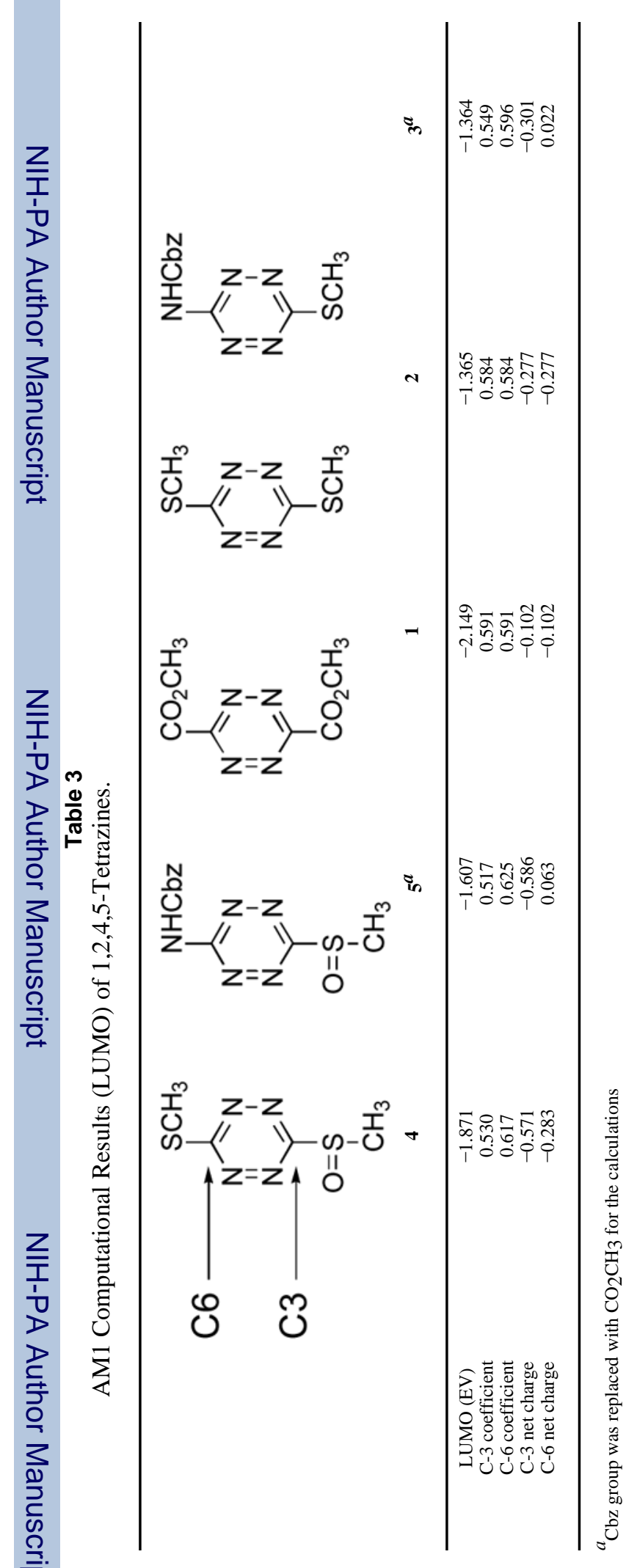

J Org Chem. Author manuscript; available in PMC 2008 August 26. 